\title{
Own-Company Stockholding and Work Effort Preferences of an Unconstrained Executive
}

\author{
Sascha Desmettre* John Gould $^{\dagger}$ Alexander Szimayer ${ }^{\ddagger}$
}

January 27, 2011

2000 MSC Subject Classification: 49L20, 91B28, 91B16

Key Words: optimal portfolio choice, executive compensation

\begin{abstract}
We develop a framework for analyzing an executive's own-company stockholding and work effort preferences. The executive, characterized by risk aversion and work effectiveness parameters, invests his personal wealth without constraint in the financial market, including the stock of his own company whose value he can directly influence with work effort. The executive's utility-maximizing personal investment and work effort strategy is derived in closed form, and a utility indifference rationale is applied to determine his required compensation. Being unconstrained by performance contracting, the executive's work effort strategy establishes a base case for theoretical or empirical assessment of the benefits or otherwise of constraining executives with performance contracting.
\end{abstract}

*Department of Financial Mathematics, Fraunhofer ITWM, Fraunhofer-Platz 1, 67663 Kaiserslautern, Germany; Center for Mathematical and Computational Modelling and Department of Mathematics, University of Kaiserslautern, Germany. Email: sascha.desmettre@itwm.fraunhofer.de. This paper is part of the PhD thesis of Sascha Desmettre.

${ }^{\dagger}$ School of Economics and Finance, Curtin University, Perth 6845, Australia. Email: j.gould@curtin.edu.au.

${ }^{\ddagger}$ Faculty of Economics and Law, University of Bonn, Adenauerallee 24-42, 53113 Bonn, Germany. Email: szimayer@uni-bonn.de. 


\section{Introduction}

Stemming from the agency theory fundamentals of Ross (1973), Jensen and Meckling (1976), Holmstrom (1979) and others, there has been much concern for the 'incentivization' link from equity-based executive compensation to corporate financial performance. The associated academic literature is extensive. ${ }^{1}$ Counterpoint to past research, we consider the motivation for an executive with unconstrained (unincentivized) compensation to voluntarily performance-link his personal wealth. We develop a model framework that identifies the joint own-company stockholding and work effort strategy of a utility-maximizing executive. The executive's compensation is assumed to be incorporated into his up-front total personal wealth, which he invests variously in a risk-free money market account, a diversified market portfolio, or his own company's stock. The executive is able to beneficially influence the value of his company via work effort; he gains utility from the increased value of his direct stockholding (within his overall personal portfolio), but loses utility for his work effort. The executive is characterized by a risk aversion parameter $(\gamma)$, and two work effectiveness parameters $(\kappa$, representing inverse work productivity, and $\alpha$, representing disutility stress).

A feature of our framework is that the executive's work effort, specified in terms of two control variables, non-systematic expected return and volatility $(\mu$ and $\sigma$ ), can be restated in terms of a single control variable, the non-systematic Sharpe ratio $(\lambda=(\mu-r) / \sigma$, where $r$ is the risk-free rate of return). This reduces the dimensions of the problem and introduces a parameterization based on the well-known Sharpe ratio performance measure. The executive's optimal personal investment and work effort strategy is then derived in closed form using stochastic control theory and the corresponding Hamilton-Jacobi-Bellman equations. Other technical papers similarly concerned with dynamic principal-agent models include Cadenillas, Cvitanic and Zapatero (2004), Korn and Kraft (2008) and Ou-Yang (2003), for example.

Our closed-form results demonstrate that an executive with superior work effectiveness (i.e. higher quality) will undertake more work effort for his company. But the extent to which any level of work effectiveness is put to use via work effort depends prominently on the executive's risk aversion; only if he has sufficiently low risk aversion to take on a substantial own-company

\footnotetext{
${ }^{1}$ The summaries of Murphy (1999) and Core, Guay and Larcker (2003) are useful references.
} 
stockholding will he have the incentive to apply substantial work effort. The results also provide guidance for identifying the executive's quality and risk aversion from demonstrated work effort. Or given identification of executive quality and risk aversion, the results indicate the own-company stockholding and work effort of an executive unconstrained by performance contracting, which establishes a base case for theoretical or empirical assessment of the benefits or otherwise of constraining the executive with performance contracting.

Freeing executives to self-incentivize may be a reasonable "path of least resistance' in the light of some recent and not so recent research. For example, Dittmann and Maug (2007) were unable to rationalize observed executive compensation. Using a 'standard' principal-agent efficient contracting model, their analysis indicated that executives should not, in general, be compensated with options, and that it would commonly be optimal for executives to use private savings to purchase additional stock in their own companies. Bettis, Bizjak and Lemmon (2001) found that high-ranking corporate insiders use collars and swaps to cover a significant proportion of their own-company stockholdings, allowing them to unwind the constraint of equity-based compensation. Ross (2004) repudiated the folklore that giving options to agents makes them more willing to take risks (also see Carpenter (2000)). And Jensen and Murphy (1990) proposed that private political forces in the managerial labor market constrain pay-performance sensitivity, leading most CEOs to hold trivial fractions of their firms' stock. However, Hall and Liebman (1998) and Core and Larcker (2002), for example, found support for a link from equity-based executive compensation to corporate performance.

Whether subject to constrained or unconstrained (i.e. incentivized or unincentivized) compensation, an executive's actualized performance incentive will reflect a total personal wealth perspective. Ofek and Yermack (2000) found that once managers reach a certain own-company ownership level, they actively rebalance their personal portfolios when awarded equity compensation. And Garvey and Milbourn (2003) found that market risk has little effect on the use of stock-based pay for the average executive, suggesting that executives can undo any undesired market exposure from their incentive contracts by adjusting their personal portfolios. We thus maximize our risk averse executive's utility with respect to his total wealth investable across his own company's stock, a diversified market portfolio and a risk-free money market account. Our approach has parallels with Jin (2002), but uses 
a continuous-time setting with arguably a more intuitively appealing specification of work effort and its disutility. Also see Cvitanic (2008) for a more general continuous-time framework emphasizing incentive effects when the executive can hedge equity-based compensation. A natural future extension for our framework is to specify a constrained executive subject to an imposed own-company stockholding representative of performance contracting, and to contrast his work effort strategy with that of our unconstrained executive.

The paper is organized as follows. Section 2 introduces the notation and terminology, and as a first result the optimality problem is reformulated and simplified. In Section 3 the Hamilton-Jacobi-Bellman equations characterizing the utility maximization problem are derived, and a closed-form solution is established. The results are illustrated in Section 4. Section 5 concludes. Technical proofs are moved to the Appendix.

\section{Notation and Set-up}

The financial market is defined on a filtered probability space $\left(\Omega, \mathcal{F}, P,\left(\mathcal{F}_{t}\right)_{t \geq 0}\right)$ satisfying the usual hypothesis and large enough to support two independent standard Brownian motions, $W^{P}=\left(W_{t}^{P}\right)_{t \geq 0}$ and $W=\left(W_{t}\right)_{t \geq 0}$, where $t$ indicates time. We consider a company executive that invests in the financial market. Specifically, the investment opportunities available to our executive are a risk-free money market account, a diversified market portfolio and his own company's stock. The risk-free money market account has the price process $B=\left(B_{t}\right)_{t \geq 0}$, with dynamics

$$
\mathrm{d} B_{t}=r B_{t} \mathrm{~d} t, \quad B_{0}=1
$$

where $r$ is the instantaneous risk-free rate of return, hence $B_{t}=e^{r t}$. The price process of the market portfolio, $P=\left(P_{t}\right)_{t \geq 0}$, follows the stochastic differential equation (SDE)

$$
\mathrm{d} P_{t}=P_{t}\left(\mu^{P} \mathrm{~d} t+\sigma^{P} \mathrm{~d} W_{t}^{P}\right), \quad P_{0} \in \mathbb{R}^{+},
$$

where $\mu^{P}$ and $\sigma^{P}$ are respectively the expected return and volatility of the market portfolio. The company's stock price process, $S^{\mu, \sigma}=\left(S_{t}^{\mu, \sigma}\right)_{t \geq 0}$, is a controlled diffusion with SDE

$$
\mathrm{d} S_{t}^{\mu, \sigma}=S_{t}^{\mu, \sigma}\left(\mu_{t} \mathrm{~d} t+\beta\left[\frac{\mathrm{d} P_{t}}{P_{t}}-r \mathrm{~d} t\right]+\sigma_{t} \mathrm{~d} W_{t}\right), S_{0}^{\mu, \sigma} \in \mathbb{R}^{+},
$$


where $\beta \in \mathbb{R}$ is the company's beta (i.e. the standardized covariance between the company's rate of return and that of the market portfolio, indicating systematic risk); $\mu$ is the company's expected rate of return for non-systematic risk (i.e. the expected return in excess of the beta-adjusted market portfolio's expected excess return); and $\sigma$ is the company's non-systematic volatility. Both $\mu$ and $\sigma$ are controlled by the executive. The company's stock price process and the market portfolio are dependent with the instantaneous correlation $\rho_{t}=\beta \sigma^{P} / \sqrt{\sigma_{t}^{2}+\left(\beta \sigma^{P}\right)^{2}}$.

The executive influences the company's stock price dynamics by choice of the control strategy $(\mu, \sigma)$, which is specified to be associated with work effort. The control strategy can be conceptualized as deriving from the executive's corporate investment or financing strategy. For example, identifying and initiating positive net present value projects and optimal debt versus equity financing entails work effort that adds value and affects volatility. Value is added if $\mu$ is greater than $r$, indicating excess return compensation for non-systematic risk. To ensure sensible solutions we require $\mu \geq r$, which effectively bars the executive from destroying company value $(\mu<r)$ and potentially profiting by shorting the company's stock.

The executive's instantaneous disutility of work effort at time $t$ is represented by the disutility rate $c\left(t, V_{t}, \mu_{t}, \sigma_{t}\right)$ for control strategy $\left(\mu_{t}, \sigma_{t}\right)$, where $V_{t}$ is the executive's wealth. We assume that the disutility rate $c:[0, T] \times$ $\mathbb{R}^{+} \times[r, \infty) \times \mathbb{R}^{+} \rightarrow \mathbb{R}_{0}^{+}$is a continuous and suitably differentiable function where $T$ is the executive's time horizon.

The executive's starting wealth inclusive of his compensation, $V_{0}>0$, is invested in the financial market. Ongoing continuous-time portfolio adjustment is assumed to be free of short-selling constraints, and to be self-financing (i.e. no funds are added to or withdrawn from the executive's portfolio). The portfolio is allocated with fraction $\pi^{P}=\left(\pi_{t}^{P}\right)_{t \geq 0}$ invested in the market portfolio, fraction $\pi^{S}=\left(\pi_{t}^{S}\right)_{t \geq 0}$ in the company's stock, and the remainder in the risk-free account. For investment strategy $\pi=\left(\pi^{P}, \pi^{S}\right)$, the executive's wealth process, $V^{\pi}=\left(V_{t}^{\pi}\right)_{t \geq 0}$, is

$$
\mathrm{d} V_{t}^{\pi}=V_{t}^{\pi}\left(\left(1-\pi_{t}^{P}-\pi_{t}^{S}\right) \frac{\mathrm{d} B_{t}}{B_{t}}+\pi_{t}^{P} \frac{\mathrm{d} P_{t}}{P_{t}}+\pi_{t}^{S} \frac{\mathrm{d} S_{t}^{\mu, \sigma}}{S_{t}^{\mu, \sigma}}\right), \quad V_{0}^{\pi} \in \mathbb{R}^{+} .
$$

The executive is assumed to maximize the expected terminal utility of his wealth for time horizon $T$, subject to some utility function which will be specified when deriving closed-form solutions. 
Assuming that the control of the company's stock price behavior $(\mu, \sigma)$ is determined exogenously, the executive's optimal investment decision is then described by

$$
\widehat{\Phi}(t, v)=\sup _{\pi \in \widehat{A}(t, v)} \mathbb{E}^{t, v}\left[U\left(V_{T}^{\pi}\right)\right], \quad(t, v) \in[0, T] \times \mathbb{R}^{+},
$$

where $\widehat{A}(t, v)$ denotes the set of all admissible portfolio strategies $\pi$ at time $t$ corresponding to portfolio value (i.e. wealth) $v=V_{t}>0, U$ is a utility function, and $\mathbb{E}^{t, v}$ denotes the expectation conditional on $t$ and $v$. See for example Korn and Korn (2001). The exogenously given control $(\mu, \sigma)$ affecting the dynamics of $S$ in (3) is suppressed in our notation.

Definition 2.1 Let $0 \leq t \leq T$, $t$ fixed. Further let $(\mu, \sigma)$ take values in $[r, \infty) \times(0, \infty)$. By $A(t, v)$ we denote the set of all admissible strategies $(\pi, \mu, \sigma)=\left(\left(\pi^{P}, \pi^{S}\right), \mu, \sigma\right)$ corresponding to portfolio value $v=V_{t}>0$ at time $t$, which are $\left\{\mathcal{F}_{u} ; t \leq u \leq T\right\}$-predictable processes, such that

(i) the company's stock price process

$$
\mathrm{d} S_{u}^{\mu, \sigma}=S_{u}^{\mu, \sigma}\left(\mu_{u} \mathrm{~d} u+\beta\left[\frac{\mathrm{d} P_{u}}{P_{u}}-r \mathrm{~d} u\right]+\sigma_{u} \mathrm{~d} W_{u}\right), \quad S_{t}^{\mu, \sigma} \in \mathbb{R}^{+},
$$

has a unique non-negative solution and satisfies

$$
\int_{t}^{T}\left(S_{u}^{\mu, \sigma}\right)^{2}\left(\left(\beta \sigma^{P}\right)^{2}+\left(\sigma_{u}\right)^{2}\right) \mathrm{d} u<\infty \quad P-\text { a.s. }
$$

(ii) the wealth equation

$$
\mathrm{d} V_{u}^{\pi}=V_{u}^{\pi}\left(\left(1-\pi_{u}^{P}-\pi_{u}^{S}\right) \frac{\mathrm{d} B_{u}}{B_{u}}+\pi_{u}^{P} \frac{\mathrm{d} P_{u}}{P_{u}}+\pi_{u}^{S} \frac{\mathrm{d} S_{u}^{\mu, \sigma}}{S_{u}^{\mu, \sigma}}\right), \quad V_{t}^{\pi} \in \mathbb{R}^{+},
$$

has a unique non-negative solution and satisfies

$$
\int_{t}^{T}\left(V_{u}^{\pi}\right)^{2}\left(\left(\pi_{u}^{P}+\beta \pi_{u}^{S}\right)^{2}\left(\sigma^{P}\right)^{2}+\left(\pi_{u}^{S} \sigma_{u}\right)^{2}\right) \mathrm{d} u<\infty \quad P-a . s .
$$

where $(\mu, \sigma)$ affects $V^{\pi}$ via $S^{\mu, \sigma}$; 
(iii) and the utility of wealth and the disutility of control satisfy

$$
\mathbb{E}\left[U\left(V_{T}^{\pi}\right)^{-}+\int_{t}^{T} c\left(u, V_{u}^{\pi}, \mu_{u}, \sigma_{u}\right) \mathrm{d} u\right]<\infty .
$$

The optimal investment and control decision is then the solution of

$$
\Phi(t, v)=\sup _{(\pi, \mu, \sigma) \in A(t, v)} \mathbb{E}^{t, v}\left[U\left(V_{T}^{\pi}\right)-\int_{t}^{T} c\left(u, V_{u}^{\pi}, \mu_{u}, \sigma_{u}\right) \mathrm{d} u\right],
$$

where $(t, v) \in[0, T] \times \mathbb{R}^{+}$.

\subsection{Restating the Set-up}

A decomposition result for the optimal investment and control problem in (6) is derived. To do this we respecify the executive's control strategy in terms of a target non-systematic Sharpe ratio $\lambda=(\mu-r) / \sigma$; this supposes the executive makes investment or financing decisions with regard for their expected return to risk trade-off. Now the original four-dimensional maximization problem can be solved in two steps. The first step entails minimizing the disutility rate for the target non-systematic Sharpe ratio to obtain $c^{\star}(t, v, \lambda)$. The proof of Lemma 2.1 demonstrates that this is achievable given Assumption 2.1. For the second step, Theorem 2.3 shows that the optimal investment and control problem given by (6) can be restated and solved as a maximization problem over the three controls $\pi^{P}, \pi^{S}$ and $\lambda$, with $c$ replaced by $c^{\star}$.

The non-systematic expected return to risk trade-off represented by $\lambda$ indicates the quality of the executive's control decision, which is associated with work effort and thereby disutility. Given $\lambda$, minimized disutility $c^{\star}$ is associated with the non-systematic volatility choice $\sigma^{\star}$ (see Lemma 2.1). That is, for a given level of control strategy quality represented by $\lambda, \sigma^{\star}$ is the non-systematic volatility associated with the 'easiest' control strategy from the executive's perspective.

Assumption 2.1 gives the conditions required for existence and uniqueness of $c^{\star}$ and $\sigma^{\star}$.

Assumption 2.1 The function $c:[0, T] \times \mathbb{R}^{+} \times[r, \infty) \times \mathbb{R}^{+} \rightarrow \mathbb{R}_{0}^{+},(t, v, \mu, \sigma)$ $\mapsto c(t, v, \mu, \sigma)$ satisfies: 
(i) $c$ is continuous in $t$ and $v$, and twice continuously differentiable in $\mu$ and $\sigma$;

(ii) fix $(t, v, \lambda) \in[0, T] \times \mathbb{R}^{+} \times \mathbb{R}_{0}^{+}$, then

$$
\limsup _{\sigma \searrow 0} \lambda \frac{\partial c}{\partial \mu}(t, v, r+\lambda \sigma, \sigma)+\frac{\partial c}{\partial \sigma}(t, v, r+\lambda \sigma, \sigma)<0,
$$

and

$$
\sup _{\sigma>0} \lambda \frac{\partial c}{\partial \mu}(t, v, r+\lambda \sigma, \sigma)+\frac{\partial c}{\partial \sigma}(t, v, r+\lambda \sigma, \sigma)>0
$$

(iii) it holds that

$$
(\mu-r)^{2} \frac{\partial^{2} c}{\partial \mu^{2}}+2 \sigma(\mu-r) \frac{\partial^{2} c}{\partial \mu \partial \sigma}+\sigma^{2} \frac{\partial^{2} c}{\partial \sigma^{2}}>0
$$

(iv) for all $(t, v): \inf _{\sigma>0} c(t, v, r, \sigma)=0$.

In Assumption 2.1, (i) is a natural smoothness condition, (ii) and (iii) respectively ensure uniqueness and existence of the disutility $c^{\star}(t, v, \lambda)$ depending on the non-systematic Sharpe ratio $\lambda$, and (iv) is a natural norming condition that specifies a lower bound of zero disutility (i.e. zero work effort) when expected excess return is zero $(\mu=r)$.

As an example, a disutility function that fulfills the conditions of Assumption 2.1 is

$$
c(t, v, \mu, \sigma)=\kappa\left(\frac{\mu-r}{\sigma}\right)^{\alpha}+\nu\left(\sigma-\sigma_{0}\right)^{2}=\kappa \lambda^{\alpha}+\nu\left(\sigma-\sigma_{0}\right)^{2},
$$

where $\mu \geq r, \sigma>0, \kappa \geq 0, \nu>0, \alpha>0$; and $\sigma_{0}>0$ is the company's base-level non-systematic risk. Here $c$ is proportional to $\lambda$ depending on parameters $\kappa$ and $\alpha$ (i.e. the executive's work effort is proportional to the quality of his control decision); and $c$ increases with deviation of control choice $\sigma$ from $\sigma_{0}$ depending on parameter $\nu$ (i.e. given $\lambda$, the executive's easiest control decision is to make investment or financing decisions that do not disrupt the company's base-level non-systematic volatility, which might be conceptualized as a preference for maintaining the status quo of the company's business model).

The following lemma establishes the first step of the decomposition result and is proved in the Appendix. 
Lemma 2.1 Suppose Assumption 2.1 holds, then the minimization problem

$$
\min _{\{\sigma>0: \mu=r+\lambda \sigma\}} c(t, v, \mu, \sigma), \quad \text { for }(t, v, \lambda) \in[0, T] \times \mathbb{R}^{+} \times \mathbb{R}_{0}^{+},
$$

admits a unique solution $\sigma^{\star}(t, v, \lambda)$.

Changing the parameters of the optimal investment and control problem in (6) from $\pi^{P}, \pi^{S}$, and $(\mu, \sigma)$ to $\pi^{P}, \pi^{S}$, and $\lambda$, and replacing $c$ by $c^{\star}$, requires adapting Def. 2.1 to the new setting. Before we present the new framework, observe that the company's stock price with respect to $\lambda$ (and $\sigma^{\star}(\lambda)$ ) has the dynamics

$$
\begin{aligned}
& \mathrm{d} S_{t}^{\lambda}=S_{t}^{\lambda}\left(\left[r+\lambda_{t} \sigma^{\star}\left(t, V_{t}^{\pi}, \lambda_{t}\right)\right] \mathrm{d} t+\beta\left[\frac{\mathrm{d} P_{t}}{P_{t}}-r \mathrm{~d} t\right]+\sigma^{\star}\left(t, V_{t}^{\pi}, \lambda_{t}\right) \mathrm{d} W_{t}\right), \\
& S_{0}^{\lambda} \in \mathbb{R}^{+} .
\end{aligned}
$$

Further, we define the minimized disutility $c^{\star}$ corresponding to portfolio value $v>0$ at time $t$ via

$$
c^{\star}(t, v, \lambda):=c\left(t, v, r+\lambda \sigma^{\star}(t, v, \lambda), \sigma^{\star}(t, v, \lambda)\right)=\min _{\{\sigma>0: \mu=r+\lambda \sigma\}} c(t, v, \mu, \sigma) .
$$

For the stock price process $S_{t}^{\lambda}$ defined in (8) we have to impose a technical condition similar to Def. 2.1 (i). The change of control from $(\mu, \sigma)$ to $\lambda$ is driven by the disutility function $c$, i.e. $\sigma^{\star}(t, v, \lambda)$ is determined by the form of $c$ (see Lemma 2.1). The following assumption guarantees that the most cost efficient strategies are admissible.

Assumption 2.2 For a given control $(\pi, \mu, \sigma) \in A(t, v)$, the value process $V_{t}^{\pi}, \lambda_{t}=\left(\mu_{t}-r\right) / \sigma_{t}$ and $\sigma^{\star}\left(t, V_{t}^{\pi}, \lambda_{t}\right)$ are determined in accordance with Lemma 2.1. The process $S_{t}^{\lambda}$ defined in (8) is assumed to satisfy

$$
\int_{t}^{T}\left(S_{u}^{\lambda}\right)^{2}\left(\left(\beta \sigma^{P}\right)^{2}+\left(\sigma_{u}^{\star}\right)^{2}\right) \mathrm{d} u<\infty \quad P-\text { a.s. }
$$


Definition 2.2 Let $0 \leq t \leq T$, $t$ fixed, and let further $\lambda$ take values in $[0, \infty)$. Then we denote by $A^{\prime}(t, v)$ the set of admissible strategies $(\pi, \lambda)=$ $\left(\left(\pi^{P}, \pi^{S}\right), \lambda\right)$ corresponding to portfolio value $v=V_{t}>0$ at time $t$, which are $\left\{\mathcal{F}_{u} ; t \leq u \leq T\right\}$-predictable processes, such that

(i) the company's stock price process

$$
\mathrm{d} S_{u}^{\lambda}=S_{u}^{\lambda}\left(\left[r+\lambda_{u} \sigma_{u}^{\star}\right] \mathrm{d} t+\beta\left[\frac{\mathrm{d} P_{u}}{P_{u}}-r \mathrm{~d} u\right]+\sigma_{u}^{\star} \mathrm{d} W_{u}\right), \quad S_{t}^{\lambda} \in \mathbb{R}^{+},
$$

has a unique non-negative solution and satisfies

$$
\int_{t}^{T}\left(S_{u}^{\lambda}\right)^{2}\left(\left(\beta \sigma^{P}\right)^{2}+\left(\sigma_{u}^{\star}\right)^{2}\right) \mathrm{d} u<\infty \quad P-a . s . ;
$$

(ii) the wealth equation

$$
\mathrm{d} V_{u}^{\pi}=V_{u}^{\pi}\left(\left(1-\pi_{u}^{P}-\pi_{u}^{S}\right) \frac{\mathrm{d} B_{u}}{B_{u}}+\pi_{u}^{P} \frac{\mathrm{d} P_{u}}{P_{u}}+\pi_{u}^{S} \frac{\mathrm{d} S_{u}^{\lambda}}{S_{u}^{\lambda}}\right), V_{t}^{\pi} \in \mathbb{R}^{+},
$$

has a unique non-negative solution and satisfies

$$
\int_{t}^{T}\left(V_{u}^{\pi}\right)^{2}\left(\left(\pi_{u}^{P}+\beta \pi_{u}^{S}\right)^{2}\left(\sigma^{P}\right)^{2}+\left(\pi_{u}^{S} \sigma_{u}^{\star}\right)^{2}\right) \mathrm{d} u<\infty \quad P-\text { a.s. }
$$

where $\lambda$ affects $V^{\pi}$ via $S^{\lambda}$;

(iii) and the utility of wealth and the minimized disutility of control satisfy

$$
\mathbb{E}\left[U\left(V_{T}^{\pi}\right)^{-}+\int_{t}^{T} c^{\star}\left(u, V_{u}^{\pi}, \lambda_{u}\right) \mathrm{d} u\right]<\infty .
$$

Theorem 2.3 (Correspondence Result) Suppose (6) admits a solution $\Phi$, then this solution coincides with the value function of the optimal investment and control problem

$\Phi^{\prime}(t, v)=\sup _{(\pi, \lambda) \in A^{\prime}(t, v)} \mathbb{E}^{t, v}\left[U\left(V_{T}^{\pi}\right)-\int_{t}^{T} c^{\star}\left(u, V_{u}^{\pi}, \lambda_{u}\right) \mathrm{d} u\right],(t, v) \in[0, T] \times \mathbb{R}^{+}$,

where $A^{\prime}(t, v)$ is given in Def. 2.2. 
Proof. Let

$$
J(t, v ; \pi, \mu, \sigma):=\mathbb{E}^{t, v}\left[U\left(V_{T}^{\pi}\right)-\int_{t}^{T} c\left(u, V_{u}^{\pi}, \mu\left(u, V_{u}^{\pi}\right), \sigma\left(u, V_{u}^{\pi}\right)\right) \mathrm{d} u\right]
$$

and

$$
J^{\prime}(t, v ; \pi, \lambda):=\mathbb{E}^{t, v}\left[U\left(V_{T}^{\pi}\right)-\int_{t}^{T} c^{\star}\left(u, V_{u}^{\pi}, \lambda\left(u, V_{u}^{\pi}\right)\right) \mathrm{d} u\right] .
$$

The assertion is proven if we show that

$$
\sup _{(\pi, \mu, \sigma) \in A(t, v)} J(t, v ; \pi, \mu, \sigma)=\sup _{(\pi, \lambda) \in A^{\prime}(t, v)} J^{\prime}(t, v ; \pi, \lambda),
$$

i.e. the performance functionals $J$ and $J^{\prime}$ admit the same value function $\Phi(t, v)$.

First, we are given controls $(\pi, \mu, \sigma) \in A(t, v)$ (and the resulting nonsystematic Sharpe ratio $\lambda=(\mu-r) / \sigma)$, and show that there exist controls $(\tilde{\pi}, \tilde{\lambda}) \in \tilde{A}(t, v)$ such that $J(t, v ; \pi, \mu, \sigma) \leq J^{\prime}(t, v ; \tilde{\pi}, \tilde{\lambda})$. Note that replacing the controls $\mu$ and $\sigma$ by $\lambda$ and replacing the disutility $c$ by $c^{\star}$ leads to two different systems of controlled SDEs describing the executive's utilitymaximizing behavior. For controls $\left(\tilde{\pi}^{P}, \tilde{\pi}^{S}, \tilde{\lambda}\right)$, we write the dynamics of the resulting price processes as follows

$$
\begin{aligned}
& \mathrm{d} \tilde{B}_{t}=\tilde{B}_{t} r \mathrm{~d} t, \mathrm{~d} \tilde{P}_{t}=\tilde{P}_{t}\left(\left[r+\lambda^{P} \sigma^{P}\right] \mathrm{d} t+\sigma^{P} \mathrm{~d} W_{t}^{P}\right), \\
& \mathrm{d} \tilde{S}_{t}=\tilde{S}_{t}\left(\left[r+\tilde{\lambda}_{t} \sigma^{\star}\left(t, \tilde{V}_{t}^{\tilde{\pi}}, \tilde{\lambda}_{t}\right)\right] \mathrm{d} t+\beta\left[\frac{\mathrm{d} \tilde{P}_{t}}{\tilde{P}_{t}}-r \mathrm{~d} t\right]+\sigma^{\star}\left(t, \tilde{V}_{t}^{\tilde{\pi}}, \tilde{\lambda}_{t}\right) \mathrm{d} W_{t}\right), \\
& \mathrm{d} \tilde{V}_{t}^{\tilde{\pi}}=\tilde{V}_{t}^{\tilde{\pi}}\left(\left[r+\tilde{\pi}_{t}^{P} \lambda^{P} \sigma^{P}+\tilde{\pi}_{t}^{S}\left(\tilde{\lambda}_{t} \sigma^{\star}\left(t, \tilde{V}_{t}^{\tilde{\pi}}, \tilde{\lambda}_{t}\right)+\beta\left(\mu^{P}-r\right)\right)\right] \mathrm{d} t\right. \\
& \left.+\tilde{\pi}_{t}^{P} \sigma^{P} \mathrm{~d} W_{t}^{P}+\tilde{\pi}_{t}^{S} \beta \sigma^{P} \mathrm{~d} W_{t}^{P}+\tilde{\pi}_{t}^{S} \sigma^{\star}\left(t, \tilde{V}_{t}^{\tilde{\pi}}, \tilde{\lambda}_{t}\right) \mathrm{d} W_{t}\right) .
\end{aligned}
$$

The system $\left(\tilde{B}, \tilde{P}, \tilde{S}, \tilde{V}^{\tilde{\pi}}\right)$ is specified on the same probability space as the original system $\left(B, P, S, V^{\pi}\right)$. We now choose the controls

$$
\tilde{\lambda}_{t}:=\lambda_{t}, \quad \tilde{\pi}_{t}^{P}:=\pi_{t}^{P}+\pi_{t}^{S} \beta\left(1-\frac{\sigma\left(t, V_{t}^{\pi}\right)}{\sigma^{\star}\left(t, \tilde{V}_{t}^{\tilde{\pi}}, \tilde{\lambda}_{t}\right)}\right), \quad \tilde{\pi}_{t}^{S}:=\pi_{t}^{S} \frac{\sigma\left(t, V_{t}^{\pi}\right)}{\sigma^{\star}\left(t, \tilde{V}_{t}^{\tilde{\pi}}, \tilde{\lambda}_{t}\right)} .
$$

This yields that the integrands of the stochastic integrals (or, coefficients of the SDEs) defining $\mathrm{d} V^{\pi}$ and $\mathrm{d} \tilde{V}^{\tilde{\pi}}$ coincide almost-surely for each $t$. Noting 
that there exist continuous versions of the resulting processes $V^{\pi}$ and $\tilde{V}^{\tilde{\pi}}$, we obtain uniformly on $[0, T]$

$$
\tilde{V}^{\tilde{\pi}}=V^{\pi}, \quad \text { and } \quad \tilde{\lambda}=\lambda, \quad P-\text { a.s. } .
$$

We remark here that by definition we also have $\tilde{B}=B$ and $\tilde{P}=P$. However, in general $\tilde{S} \neq S$. Continuing the proof, by $c^{\star}(t, v, \lambda):=c(t, v, r+$ $\left.\lambda \sigma^{\star}(t, v, \lambda), \sigma^{\star}(t, v, \lambda)\right)=\min _{\{\sigma>0: \mu=r+\lambda \sigma\}} c(t, v, \mu, \sigma)$ and recalling that $\lambda=$ $(\mu-r) / \sigma$ we have:

$$
\begin{aligned}
J(t, v ; \pi, \mu, \sigma) & \leq \mathbb{E}^{t, v}\left[U\left(V_{T}^{\pi}\right)-\int_{t}^{T} c^{\star}\left(u, V_{u}^{\pi}, \lambda\right) \mathrm{d} u\right] \\
& \stackrel{(12)}{=} \mathbb{E}^{t, v}\left[U\left(\tilde{V}_{T}^{\tilde{\pi}}\right)-\int_{t}^{T} c^{\star}\left(u, \tilde{V}_{u}^{\tilde{\pi}}, \tilde{\lambda}_{u}\right) \mathrm{d} u\right]=J^{\prime}(t, v ; \tilde{\pi}, \tilde{\lambda}) .
\end{aligned}
$$

To finish the first part of the proof, we have to ensure $(\tilde{\pi}, \tilde{\lambda}) \in A^{\prime}(t, v)$. This can be done by recalling that $(\pi, \mu, \sigma) \in A(t, v)$ and checking conditions (i),(ii) and (iii) of Def. 2.2. First note that (i) is satisfied due to Assumption 2.2. To verify (ii) note that

$$
\begin{aligned}
\tilde{V}_{u}^{\tilde{\pi}} & =V_{u}^{\pi}, \quad P-\text { a.s. } \\
\tilde{\pi}_{u}^{P}+\beta \tilde{\pi}_{u}^{S} & =\pi_{u}^{P}+\beta \pi_{u}^{S}, \quad P-\text { a.s. } \\
\tilde{\pi}_{u}^{S} \sigma^{\star}\left(u, \tilde{V}_{u}^{\tilde{\pi}}, \tilde{\lambda}_{u}\right) & =\pi_{u}^{S} \sigma_{u}, \quad P-\text { a.s. }
\end{aligned}
$$

for $t \leq u \leq T$, and recall Def. 2.1 (ii). To verify (iii), check that $c_{u}^{\star}\left(u, \tilde{V}^{\tilde{\pi}}, \tilde{\lambda}_{u}\right) \leq$ $c\left(u, V^{\pi}, \mu_{u}, \sigma_{u}\right)$, for $t \leq u \leq T$, and then recall Def. 2.1 (iii) to obtain an integrable upper bound.

To conclude the proof we have to show that for a given control $(\tilde{\pi}, \tilde{\lambda}) \in$ $A^{\prime}(t, v)$ there is a corresponding control $(\pi, \mu, \sigma) \in A(t, v)$ s.t. $J^{\prime}(t, v ; \tilde{\pi}, \tilde{\lambda})$ $\leq J(t, v ; \pi, \mu, \sigma)$. To do so, set $\sigma_{u}=\sigma^{\star}\left(u, \tilde{V}_{u}^{\tilde{\pi}}, \tilde{\lambda}_{u}\right), \mu_{u}=r+\tilde{\lambda}_{u} \sigma_{u}$, and $\tilde{\pi}_{u}=\pi_{u}$, for $t \leq u \leq T$, to obtain $J^{\prime}(t, v ; \tilde{\pi}, \tilde{\lambda})=J(t, v ; \pi, \mu, \sigma)$. Finally, $(\pi, \mu, \sigma) \in A(t, v)$ is verified directly by checking Def. 2.1 using $(\tilde{\pi}, \tilde{\lambda}) \in$ $A^{\prime}(t, v)$ and Def. 2.2.

\section{Optimal Strategies}

In this section we use stochastic control techniques to derive closed-form solutions to the investment and control decision problem in (10), for special 
choices of the utility and disutility functions. In particular we specify constant relative risk aversion. For the relative risk aversion parameter $\gamma>0$, the utility function $U$ is

$$
U(v)= \begin{cases}\frac{v^{1-\gamma}}{1-\gamma}, & \text { for } \gamma>0 \text { and } \gamma \neq 1 \\ \log (v), & \text { for } \gamma=1\end{cases}
$$

and the disutility of control (i.e. work effort) $c^{\star}$ is

$$
c^{\star}(t, v, \lambda)=\kappa v^{1-\gamma} \frac{\lambda^{\alpha}}{\alpha}, \quad \gamma>0,
$$

where $\kappa>0$ and $\alpha>2$ are the executive's work effectiveness parameters, respectively termed 'inverse work productivity' and 'disutility stress'. $\kappa$ directly relates the executive's work effort disutility to the quality of his control decision as indicated by the non-systematic Sharpe ratio $\lambda$, and $\alpha$ indicates how rapidly his work effort disutility will rise for the sake of an improved $\lambda$. The requirement $\alpha>2$ is a consequence of our set-up that ensures the executive's disutility grows with work effort, i.e. $\lambda$, at a rate that offsets (at some level of $\lambda$ ) the rate of his utility gain due to the flow-on from his work effort to the value of his own-company stockholding; this becomes evident with derivation of the solution to (10). A higher quality executive is able to achieve a given $\lambda$ with lower disutility, and is able to improve $\lambda$ with lower incremental disutility. That is, higher executive quality (i.e. higher work effectiveness) is implied by lower values of $\kappa$ and $\alpha$.

In (14), the scaling factor $v^{1-\gamma}$ relates the executive's disutility of work effort to his wealth $(v)$ with a formulation based on the constant relative risk aversion formulation of the utility function in (13). Given a low (high) value of the relative risk aversion parameter, $0<\gamma<1(\gamma>1)$, the executive's work effort disutility increases (decreases) with his wealth at a decreasing rate; and for $\gamma=1$, work effort disutility is unrelated to wealth.

Remark 3.1 Our specification for the disutility of work effort is economically reasonable for the case $0<\gamma<1$. For $\gamma>1$, our specification produces decreasing disutility of work effort for an increasing level of wealth, keeping work effort constant. This is economically counter-intuitive. Nevertheless, we solve our executive's investment and control problem for all values of $\gamma>0$. 
A possible rationalization is to consider $\gamma$ to be positively related to the executive's work ethic, such that a high work ethic executive has comparatively low aversion to work effort at outset and will become further less averse to work effort if past effort or chance brings success as indicated by increased wealth. Whereas a low work ethic executive has comparatively high aversion to work effort and will become further more averse to work effort if his wealth increases.

For the remainder of the paper we assume that the optimal investment and control problem (10) admits a value function $\Phi \in C^{1,2}$.

To guarantee that the candidates we will derive for the executive's optimal investment and control strategy (i.e. the choices for own-company stockholding, market portfolio holding and non-systematic Sharpe ratio) and value function are indeed optimal, we have to consider a more restrictive class of admissible strategies as follows.

Definition 3.1 Let $0 \leq t \leq T$, $t$ fixed, and let $\lambda$ take values in $[0, \infty)$. Then by $A_{\gamma}^{\prime}(t, v)$ we denote the set of admissible strategies $(\pi, \lambda) \in A^{\prime}(t, v)$, such that

(i) for $\gamma>0$ and $\gamma \neq 1$ :

$$
\begin{aligned}
& \int_{t}^{T}\left(\pi_{u}^{P}+\beta \pi_{u}^{S}\right)^{4}\left(\sigma^{P}\right)^{4}+\left(\pi_{u}^{S} \sigma_{u}^{\star}\right)^{4} \mathrm{~d} u \leq C_{1}<\infty, \text { for some } C_{1} \in \mathbb{R}_{0}^{+} \\
& \int_{t}^{T}\left|\pi_{u}^{S} \sigma_{u}^{\star} \lambda_{u}\right| \mathrm{d} u \leq C_{2}<\infty, \text { for some } C_{2} \in \mathbb{R}_{0}^{+}
\end{aligned}
$$

(ii) for $\gamma=1$ :

$$
\mathbb{E}\left[\int_{t}^{T}\left(\pi_{u}^{P}+\beta \pi_{u}^{S}\right)^{2}\left(\sigma^{P}\right)^{2}+\left(\pi_{u}^{S} \sigma_{u}^{\star}\right)^{2} \mathrm{~d} u\right]<\infty
$$

Restating the optimal investment and control problem:

$$
\Phi(t, v)=\sup _{(\pi, \lambda) \in A_{\gamma}^{\prime}(t, v)} \mathbb{E}^{t, v}\left[U\left(V_{T}^{\pi}\right)-\int_{t}^{T} c^{\star}\left(u, V_{u}^{\pi}, \lambda_{u}\right) \mathrm{d} u\right],
$$

where $(t, v) \in[0, T] \times \mathbb{R}^{+}$.

Remark 3.2 One directly sees that $A_{\gamma}^{\prime}(t, v)$ is a subset of $A^{\prime}(t, v)$. Therefore the results previously derived for $A^{\prime}(t, v)$ remain valid for $A_{\gamma}^{\prime}(t, v)$. 


\subsection{Hamilton-Jacobi-Bellman Equation}

Having formulated the optimal investment and control decision problem with respect to the parameter set $(\pi, \lambda)$ as given by (18), we can write down the corresponding Hamilton-Jacobi-Bellman equation (HJB); note that we formulate this equation with respect to a general utility function $U$ and a general disutility function $c^{\star}$ :

$$
\begin{aligned}
0 & =\sup _{(\pi, \lambda) \in \mathbb{R}^{2} \times[0, \infty)}\left[\left(L^{(\pi, \lambda)} \Phi\right)(t, v)-c^{\star}(t, v, \lambda)\right], \text { for }(t, v) \in[0, T) \times \mathbb{R}^{+}, \\
U(v) & =\Phi(T, v), \quad \text { for } v \in \mathbb{R}^{+},
\end{aligned}
$$

where the differential operator $L^{(\pi, \lambda)}$ is given by

$$
\begin{aligned}
& \left(L^{\pi, \lambda} g\right)(t, v)=\frac{\partial g}{\partial t}(t, v)+\frac{\partial g}{\partial v}(t, v) v\left(r+\pi^{S} \lambda \sigma^{\star}(t, v, \lambda)+\pi^{S} \beta\left[\mu^{P}-r\right]\right. \\
& \left.+\pi^{P}\left[\mu^{P}-r\right]\right)+\frac{1}{2} \frac{\partial^{2} g}{\partial v^{2}}(t, v) v^{2}\left(\left[\pi^{S} \sigma^{\star}(t, v, \lambda)\right]^{2}+\left[\left(\pi^{P}+\pi^{S} \beta\right) \sigma^{P}\right]^{2}\right) .
\end{aligned}
$$

Potential maximizers $\pi^{P^{\star}}, \pi^{S^{\star}}$ and $\lambda^{\star}$ of the HJB (19) can be calculated by establishing the first order conditions:

$$
\begin{aligned}
& \pi^{P^{\star}}(t, v)=-\frac{\left(\mu^{P}-r\right)}{v\left(\sigma^{P}\right)^{2}} \frac{\Phi_{v}(t, v)}{\Phi_{v v}(t, v)}-\beta \pi^{S^{\star}}(t, v), \\
& \pi^{S^{\star}}(t, v)=-\frac{\lambda^{\star}(t, v)}{v \sigma^{\star}\left(t, v, \lambda^{\star}(t, v)\right)} \frac{\Phi_{v}(t, v)}{\Phi_{v v}(t, v)},
\end{aligned}
$$

where $\lambda^{\star}$ is the solution of the implicit equation

$$
\lambda \frac{\Phi_{v}^{2}(t, v)}{\Phi_{v v}(t, v)}+c_{\lambda}^{\star}(t, v, \lambda)=0 \quad \text { for all }(t, v) \in[0, T] \times \mathbb{R}^{+},
$$

where we have already used (21) to simplify the equation.

From (21), the executive's optimal wealth allocation to his own company's stock $\pi^{S^{\star}}$ depends on his optimal control decision for the stock price dynamics $\lambda^{\star}$. However, the executive's overall preference for investment exposure to systematic risk is independent of $\lambda^{\star}$. Therefore his optimal wealth allocation to the market portfolio $\pi^{P^{\star}}$ incorporates a deduction for the systematic risk 
exposure entailed by $\pi^{S^{\star}}$; because of this, $\pi^{P^{\star}}$ also depends on $\lambda^{\star}$ via $\pi^{S^{\star}}$ factored by the company's beta $\beta$.

Substituting the maximizers (21) in the HJB (19) yields:

$$
\begin{aligned}
0= & \Phi_{t}(t, v)+\Phi_{v}(t, v) v r-\frac{1}{2}\left(\lambda^{\star}(t, v)\right)^{2} \frac{\Phi_{v}^{2}(t, v)}{\Phi_{v v}(t, v)}-\frac{1}{2}\left(\lambda_{P}\right)^{2} \frac{\Phi_{v}^{2}(t, v)}{\Phi_{v v}(t, v)} \\
& -c^{\star}\left(t, v, \lambda^{\star}(t, v)\right),
\end{aligned}
$$

where $\lambda_{P}:=\frac{\mu^{P}-r}{\sigma^{P}}$ is the Sharpe ratio of the market portfolio.

In the following section we solve (23) with choices (13) and (14) for the utility and disutility functions.

\subsection{Closed-Form Solutions}

Closed-form solutions are obtained for the optimal investment and control problem in (18) using the utility and disutility functions (13) and (14), first for the power-utility case $(\gamma>0$ and $\gamma \neq 1)$, and then for the log-utility case $(\gamma=1)$.

Theorem 3.1 (The power-utility case: $\gamma>0$ and $\gamma \neq 1$ ) The full solution of the maximization problem (18) can be summarized by the strategy

$$
\begin{aligned}
& \lambda^{\star}(t, v)=\left(\frac{1}{\kappa \gamma} f(t)\right)^{\frac{1}{\alpha-2}} \\
& \pi^{P^{\star}}(t, v)=\frac{\mu^{P}-r}{\gamma\left(\sigma^{P}\right)^{2}}-\beta \pi^{S^{\star}}(t, v), \quad \pi^{S^{\star}}(t, v)=\frac{\lambda^{\star}(t, v)}{\gamma \sigma^{\star}\left(t, v, \lambda^{\star}(t, v)\right)},
\end{aligned}
$$

and value function

$$
\Phi(t, v)=\frac{v^{1-\gamma}}{1-\gamma} f(t)
$$

where

$$
\begin{aligned}
f(t)= & e^{(1-\gamma)\left(r+\frac{1}{2} \frac{\lambda_{P}^{2}}{\gamma}\right)(T-t)} \\
& \times\left(1-\frac{(\alpha-2)\left(\frac{1}{\kappa \gamma}\right)^{\frac{2}{\alpha-2}}}{\alpha\left(2 \gamma r+\lambda_{P}^{2}\right)}\left(e^{\frac{1-\gamma}{\alpha-2}\left(2 r+\frac{\lambda_{P}^{2}}{\gamma}\right)(T-t)}-1\right)\right)^{-\frac{\alpha-2}{2}} .
\end{aligned}
$$


Proof. First observe that a function $F$ of the form $F(\lambda)=a \lambda^{2}-b \lambda^{\alpha}$, $\lambda \geq 0$, for given constants $a, b>0$ and $\alpha>2$, has a unique maximizer $\lambda^{\star}$ and maximized value $F\left(\lambda^{\star}\right)$ given by

$$
\lambda^{\star}=\left(\frac{2 a}{\alpha b}\right)^{\frac{1}{\alpha-2}}, \quad \text { and } \quad F\left(\lambda^{\star}\right)=(\alpha-2) \alpha^{-\frac{\alpha}{\alpha-2}} 2^{\frac{2}{\alpha-2}} a^{\frac{\alpha}{\alpha-2}} b^{-\frac{2}{\alpha-2}} .
$$

Using this insight the first order condition for $\lambda^{\star}$ in $(22)$ is now solved. Set

$$
a=\frac{1}{2} \frac{\Phi_{v}^{2}}{-\Phi_{v v}}, \quad \text { and } \quad b=\frac{\kappa}{\alpha} v^{1-\gamma},
$$

then (27) gives

$$
\lambda^{\star}=\left(\frac{1}{\kappa v^{1-\gamma}} \frac{\Phi_{v}^{2}}{-\Phi_{v v}}\right)^{\frac{1}{\alpha-2}}, \quad F\left(\lambda^{\star}\right)=\frac{\alpha-2}{2 \alpha}\left(\kappa v^{1-\gamma}\right)^{-\frac{2}{\alpha-2}}\left(\frac{\Phi_{v}^{2}}{-\Phi_{v v}}\right)^{\frac{\alpha}{\alpha-2}} .
$$

Now (23) reads

$$
0=\Phi_{t}+\Phi_{v} v r+\frac{1}{2} \frac{\Phi_{v}^{2}}{-\Phi_{v v}}\left(\frac{\mu^{P}-r}{\sigma^{P}}\right)^{2}+\frac{\alpha-2}{2 \alpha}\left(\kappa v^{1-\gamma}\right)^{-\frac{2}{\alpha-2}}\left(\frac{\Phi_{v}^{2}}{-\Phi_{v v}}\right)^{\frac{\alpha}{\alpha-2}} .
$$

Using the separation ansatz $\Phi(t, v)=f(t) \frac{v^{1-\gamma}}{1-\gamma}$ results in

$$
\Phi_{t}=\dot{f} \frac{v^{1-\gamma}}{1-\gamma}, \quad \Phi_{v}=f v^{-\gamma}, \quad \Phi_{v v}=-\gamma f v^{-\gamma-1}, \quad \text { and } \quad f(T)=1
$$

Thus (28) becomes

$$
\begin{aligned}
0= & \dot{f} \frac{v^{1-\gamma}}{1-\gamma}+f v^{1-\gamma} r+\frac{1}{2} \frac{f v^{1-\gamma}}{\gamma}\left(\frac{\mu^{P}-r}{\sigma^{P}}\right)^{2} \\
& +\frac{\alpha-2}{2 \alpha}\left(\kappa v^{1-\gamma}\right)^{-\frac{2}{\alpha-2}}\left(\frac{f v^{1-\gamma}}{\gamma}\right)^{\frac{\alpha}{\alpha-2}} .
\end{aligned}
$$

Dividing by $\frac{v^{1-\gamma}}{1-\gamma}$ and recalling $\lambda_{P}=\left(\mu^{P}-r\right) / \sigma^{P}$ gives

$$
\dot{f}=f\left[-(1-\gamma)\left(r+\frac{1}{2} \frac{\lambda_{P}^{2}}{\gamma}\right)\right]+f^{\frac{\alpha}{\alpha-2}}\left[-(1-\gamma) \frac{\kappa}{2} \frac{\alpha-2}{\alpha}\left(\frac{1}{\kappa \gamma}\right)^{\frac{\alpha}{\alpha-2}}\right] \text {. }
$$


This is a Bernoulli ordinary differential equation (ODE) of the form $\dot{f}=$ $a_{1} f+a_{\nu} f^{\nu}$, with solution

$$
f(t)^{1-\nu}=C e^{G(t)}+(1-\nu) e^{G(t)} \int_{0}^{t} e^{-G(s)} a_{\nu} \mathrm{d} s,
$$

where $G(t)=(1-\nu) \int_{0}^{t} a_{1}(s) \mathrm{d} s$ and $C$ is an arbitrary constant. In our setting we have $\nu=\frac{\alpha}{\alpha-2}$ and $(1-\nu)=\frac{-2}{\alpha-2}$ implying

$$
a_{1}=-(1-\gamma)\left(r+\frac{1}{2} \frac{\lambda_{P}^{2}}{\gamma}\right), a_{\nu}=-(1-\gamma) \frac{\kappa}{2} \frac{\alpha-2}{\alpha}\left(\frac{1}{\kappa \gamma}\right)^{\frac{\alpha}{\alpha-2}} .
$$

The formal solution $f(t)^{1-\nu}$ is explicitly calculated in three steps. First, compute

$$
G(t)=-\frac{2 a_{1} t}{\alpha-2}, \quad \text { and } \quad \int_{0}^{t} e^{-G(s)} a_{\nu}(s) \mathrm{d} s=\frac{\alpha-2}{2} \frac{a_{\nu}}{a_{1}}\left(e^{\frac{2 a_{1} t}{\alpha-2}}-1\right),
$$

then

$$
f(t)=e^{a_{1} t}\left(C-\frac{a_{\nu}}{a_{1}}\left(e^{\frac{2 a_{1} t}{\alpha-2}}-1\right)\right)^{-\frac{\alpha-2}{2}} .
$$

Finally, solve for $C$ by using $f(T)=1$ so that

$$
C=e^{\frac{2 a_{1} T}{\alpha-2}}+\frac{a_{\nu}}{a_{1}}\left(e^{\frac{2 a_{1} T}{\alpha-2}}-1\right) .
$$

Note also that $f(0)=C^{-\frac{\alpha-2}{2}}$. Now

$$
f(t)=e^{-a_{1}(T-t)}\left(1-\frac{a_{\nu}}{a_{1}}\left(e^{-\frac{2 a_{1}}{\alpha-2}(T-t)}-1\right)\right)^{-\frac{\alpha-2}{2}} .
$$

Substituting for $a_{1}$ and $a_{\nu}$ then yields the result for $f(t)$. Using $\frac{\Phi_{v}}{\Phi_{v v}}=-\frac{v}{\gamma}$ and the first order conditions in (21) we obtain the claimed optimal strategy $\lambda^{\star}$, $\pi^{P^{\star}}$ and $\pi^{S^{\star}}$. Finally note that our claimed optimal strategies are admissible, i.e. $\left(\pi^{S^{\star}}, \pi^{P^{\star}}, \lambda^{\star}\right) \in A_{\gamma}^{\prime}(t, v)$. A sufficient condition for admissibility is that $\lambda^{\star}, \pi^{P^{\star}} \sigma^{P}$, and $\pi^{S^{\star}} \sigma^{\star}$ be uniformly bounded (see Def. 3.1 ); because these expressions are deterministic and continuous functions in $u$ on $[t, T]$, they are hence uniformly bounded. 
Theorem 3.2 (The log-utility case: $\gamma=1$ ) The full solution of the maximization problem (18) can be summarized by the strategy

$$
\begin{aligned}
& \lambda^{\star}(t, v)=\kappa^{-\frac{1}{\alpha-2}}, \\
& \pi^{P^{\star}}(t, v)=\frac{\mu^{P}-r}{\left(\sigma^{P}\right)^{2}}-\beta \pi^{S^{\star}}(t, v), \quad \pi^{S^{\star}}(t, v)=\frac{\lambda^{\star}(t, v)}{\sigma^{\star}\left(t, v, \lambda^{\star}(t, v)\right)},
\end{aligned}
$$

and value function

$$
\Phi(t, v)=\log (v)+\left[r+\frac{1}{2}\left(\frac{\mu^{P}-r}{\sigma^{P}}\right)^{2}+\frac{\alpha-2}{2 \alpha} \kappa^{-\frac{2}{\alpha-2}}\right](T-t) .
$$

Proof. As in the power-utility case, first the implicit first order condition for $\lambda^{\star}$ in (22) is made explicit. This time set

$$
a=\frac{1}{2} \frac{\Phi_{v}^{2}}{-\Phi_{v v}}, \quad \text { and } \quad b=\frac{\kappa}{\alpha}
$$

then (27) gives

$$
\lambda^{\star}=\left(\frac{1}{\kappa} \frac{\Phi_{v}^{2}}{-\Phi_{v v}}\right)^{\frac{1}{\alpha-2}}, \text { and } F\left(\lambda^{\star}\right)=\frac{\alpha-2}{2 \alpha} \kappa^{-\frac{2}{\alpha-2}}\left(\frac{\Phi_{v}^{2}}{-\Phi_{v v}}\right)^{\frac{\alpha}{\alpha-2}} .
$$

The partial differential equation (PDE) for log-utility now reads

$$
0=\Phi_{t}+\Phi_{v} v r+\frac{1}{2} \frac{\Phi_{v}^{2}}{-\Phi_{v v}}\left(\frac{\mu^{P}-r}{\sigma^{P}}\right)^{2}+\frac{\alpha-2}{2 \alpha} \kappa^{-\frac{2}{\alpha-2}}\left(\frac{\Phi_{v}^{2}}{-\Phi_{v v}}\right)^{\frac{\alpha}{\alpha-2}} .
$$

Using the ansatz $\Phi(t, v)=\log (v)+\varphi(T-t)$ results in

$$
\Phi_{t}=-\varphi, \quad \Phi_{v}=\frac{1}{v}, \quad \Phi_{v v}=-\frac{1}{v^{2}}, \quad \text { and } \quad \Phi(T, v)=\log (v)=U(v) .
$$

Then (33) reduces to

$$
\varphi=r+\frac{1}{2}\left(\frac{\mu^{P}-r}{\sigma^{P}}\right)^{2}+\frac{\alpha-2}{2 \alpha} \kappa^{-\frac{2}{\alpha-2}} .
$$

Finally, noting $\Phi_{v}^{2} / \Phi_{v v}=-1$ and using the first order conditions in (21) establishes the claimed optimal strategy. Using identical rationale as in the proof of Theorem 3.1, we see that $\left(\pi^{S^{\star}}, \pi^{P^{\star}}, \lambda^{\star}\right) \in A_{1}^{\prime}(t, v)$. Note that we also obtain the form of the optimal strategy by formally setting $\gamma=1$ in Theorem 3.1. 


\subsection{Verification Theorem}

The solutions of the maximization problems given in Theorems 3.1 and 3.2 are candidates for the optimal investment and control choices for the problem in (18). In this section we verify that under sufficient assumptions these solutions are indeed optimal.

Theorem 3.3 (Verification Result) Let $\kappa>0$ and $\alpha>2$. Assume the executive's utility and disutility functions are given by (13) and (14). Then the candidates given in (24) - (26) are the optimal investment and control strategy (i.e. own-company stockholding, market portfolio holding and nonsystematic Sharpe ratio strategy) and value function of the optimal control problem (18) for the case $\gamma>0$ and $\gamma \neq 1$; and the candidates given in (31) and (32) are the optimal investment and control strategy and value function of the optimal control problem (18) for the case $\gamma=1$.

The proof of Theorem 3.3 is given in the Appendix.

\section{Discussion and Implications of Results}

Theorems 3.1, 3.2 and 3.3 indicate our unconstrained executive's maximized utility and associated optimal behavior in terms of personal portfolio selection and choice of work effort, subject to the constant relative risk aversion set-up. We now investigate the sensitivity of this optimal behavior to variation of the executive's risk aversion and work effectiveness characteristics. Additionally, we derive the fair compensation for the executive's work effort using a utility indifference approach (following the vein of, for example, Lambert, Larcker and Verrecchia (1991)).

The executive is characterized by the relative risk aversion coefficient $(\gamma>0)$, and two work effectiveness parameters: work productivity $(1 / \kappa$, with $\kappa>0)$, and disutility stress $(\alpha>2)$. To produce results that have relativity to a base-level of work effort, as indicated by a base-level nonsystematic Sharpe ratio control decision $\lambda_{0}>0$, the disutility $c^{\star}$ given by (14) is reparameterized so that the utility/disutility set-up becomes

$$
U(v)= \begin{cases}\frac{v^{1-\gamma}}{1-\gamma}, & \text { for } \gamma>0 \text { and } \gamma \neq 1 \\ \log (v), & \text { for } \gamma=1\end{cases}
$$


and

$$
c^{\star}(t, v, \lambda)=\frac{\kappa}{\alpha} v^{1-\gamma}\left(\frac{\lambda}{\lambda_{0}}\right)^{\alpha}, \quad \text { for } \lambda \geq 0, \quad \gamma>0 .
$$

In regard to the executive's optimal personal investment decision $\pi^{\star}$, the optimal own-company stockholding $\pi^{S^{\star}}$ is a function of the optimal work effort choice, and the associated optimal volatility $\sigma^{\star}$ (see Lemma 2.1) which we do not explicitly specify. The optimal market portfolio allocation $\pi^{P^{\star}}$ considered in conjunction with the systematic risk exposure associated with $\pi^{S^{\star}}$ coincides with the result from classical utility maximization in the constant relative risk aversion setting, and is therefore of limited interest.

We now turn to the relationship between the executive's optimal work effort/control choice $\lambda^{\star}$, his characteristics $1 / \kappa$ and $\alpha$, and his utility indifference compensation, for log-utility and power-utility cases. It is worth reiterating that $\kappa(1 / \kappa)$ directly (inversely) relates the executive's work effort disutility to the quality of his control decision as indicated by the nonsystematic Sharpe ratio $\lambda$, and $\alpha$ indicates how rapidly his work effort disutility will rise for the sake of an improved $\lambda$.

\subsection{The Log-Utility Case}

With assumption of log-utility $(\gamma=1)$, the executive's optimal choice of work effort for the new disutility parameterization is $\lambda^{\star}=\lambda_{0}^{\frac{\alpha}{\alpha-2}}(1 / \kappa)^{\frac{1}{\alpha-2}}$ (see Theorem 3.2 for the optimal choice under the original parameterization). We assume work productivity satisfies $1 / \kappa>\lambda_{0}^{-2}$ to ensure optimal work effort is not less than base-level, i.e. $\lambda^{\star} \geq \lambda_{0}>0$. Consequently, for $\lambda^{\star}=\lambda^{\star}(1 / \kappa, \alpha)$, the optimal work effort sensitivities to the work effectiveness parameters are

$$
\frac{\partial \lambda^{\star}}{\partial(1 / \kappa)}=\frac{\kappa}{\alpha-2} \lambda^{\star}>0, \frac{\partial \lambda^{\star}}{\partial \alpha}=-\frac{\ln \left(\frac{1 / \kappa}{\lambda_{0}^{-2}}\right)}{(\alpha-2)^{2}} \lambda^{\star}<0 \text {, for } \alpha>2 \text { and } 1 / \kappa>\lambda_{0}^{-2} \text {. }
$$

That is, the executive's optimal work effort choice is positively related to his work productivity $\left(\partial \lambda^{\star} / \partial(1 / \kappa)>0\right)$, and negatively related to his disutility stress $\left(\partial \lambda^{\star} / \partial \alpha<0\right)$. This result is illustrated by Figure 1, which graphs optimal work effort versus work productivity and disutility stress, with $\lambda_{0}=0.10$. Furthermore Figure 1 indicates that, for moderate and large values of disutility stress $\alpha$, optimal work effort is mainly driven by work productivity $1 / \kappa$; 
and optimal work effort is most sensitive to low values of work productivity close to the boundary value $\left(1 / \kappa \gtrsim \lambda_{0}^{-2}=100\right)$.

The limiting cases for work productivity are

$$
\lim _{1 / \kappa \searrow \lambda_{0}^{-2}} \lambda^{\star}(1 / \kappa, \alpha)=\lambda_{0} \quad \text { and } \quad \lim _{1 / \kappa \nearrow \infty} \lambda^{\star}(1 / \kappa, \alpha)=+\infty, \quad \text { for all } \alpha>2,
$$

indicating that the limit for deteriorating work productivity is base-level work effort $\lambda_{0}$, and ever increasing work productivity yields ever increasing work effort (to infinity).

Taking disutility stress to its limiting cases gives

$$
\lim _{\alpha \searrow 2} \lambda^{\star}(1 / \kappa, \alpha)=+\infty \quad \text { and } \quad \lim _{\alpha \nearrow \infty} \lambda^{\star}(1 / \kappa, \alpha)=\lambda_{0}, \quad \text { for all } 1 / \kappa>\lambda_{0}^{-2},
$$

indicating that the executive will deliver ever increasing work effort as disutility stress diminishes, and the totally stressed executive will deliver base-level work effort.

The value function specifying the executive's maximized utility can be written as the difference between the utility from his optimal personal investment decision and the disutility from his optimal work effort (see Theorem 3.2 for the value function under the original disutility parameterization):

$$
\Phi(0, v)=\underbrace{\log (v)+\left[r+\frac{1}{2}\left(\lambda^{P}\right)^{2}+\frac{1}{2}\left(\lambda^{\star}\right)^{2}\right]}_{=\mathbb{E}^{0, v}\left[U\left(V_{T}^{\pi^{\star}}\right)\right]}-\underbrace{\frac{1}{\alpha}\left(\lambda^{\star}\right)^{2} T}_{=\mathbb{E}^{0, v} \int_{0}^{T} c^{\star}\left(t, V_{t}^{\pi^{\star}}, \lambda^{\star}\left(t, V_{t}^{\pi^{\star}}\right)\right) \mathrm{d} t} .
$$

We assume that the executive's fair compensation for the disutility of work effort is paid up-front with cash or marketable (unconstrained) securities of value $\Delta v$. Applying a utility indifference argument, the fair level of compensation satisfies

$$
\Phi(0, v+\Delta v)=\Phi(0, v)+\mathbb{E}^{0, v}\left[\int_{0}^{T} c^{\star}\left(t, V_{t}^{\pi^{\star}}, \lambda^{\star}\left(t, V_{t}^{\pi^{\star}}\right)\right) \mathrm{d} t\right],
$$

which gives

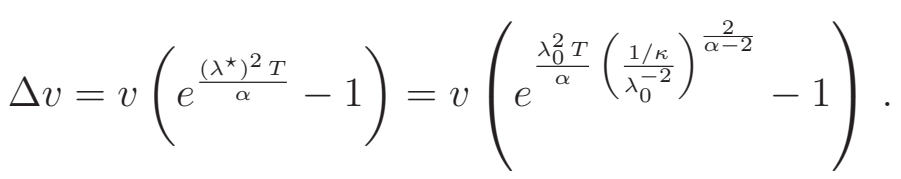


For $\Delta v=\Delta v(1 / \kappa, \alpha)$, the utility indifference compensation sensitivities to the work effectiveness parameters are

$$
\frac{\partial \Delta v}{\partial(1 / \kappa)}=\frac{2 \kappa}{\alpha-2} \frac{\left(\lambda^{\star}\right)^{2} T}{\alpha}(\Delta v+v) \quad>\quad 0, \quad \text { for } \alpha>2 \text { and } 1 / \kappa>\lambda_{0}^{-2},
$$

and

$\frac{\partial \Delta v}{\partial \alpha}=-\left(\frac{1}{\alpha}+\frac{2 \ln \left(\frac{1 / \kappa}{\lambda_{0}^{-2}}\right)}{(\alpha-2)^{2}}\right) \frac{\left(\lambda^{\star}\right)^{2} T}{\alpha}(\Delta v+v)<0$, for $\alpha>2$ and $1 / \kappa>\lambda_{0}^{-2}$,

indicating the sensible result that the executive's utility indifference compensation increases with work productivity and decreases with disutility stress. This result is illustrated by Figure 2, which graphs the executive's fair upfront compensation, based on the utility indifference rationale, versus work productivity and disutility stress, for the case where the executive's initial wealth is $v=\$ 5$ million, time horizon is $T=10$ years, and base-level work effort is $\lambda_{0}=0.10$.

The limiting cases for work productivity are

$$
\lim _{1 / \kappa \searrow \lambda_{0}^{-2}} \Delta v(1 / \kappa, \alpha)=v\left(e^{\frac{\lambda_{0}^{2} T}{\alpha}}-1\right), \quad \text { and } \lim _{1 / \kappa \nearrow^{\infty}} \Delta v(1 / \kappa, \alpha)=+\infty,
$$

for all $\alpha>2$, and the limiting cases for disutility stress are

$$
\lim _{\alpha \searrow 2} \Delta v(1 / \kappa, \alpha)=+\infty, \quad \text { and } \quad \lim _{\alpha \nearrow \infty} \Delta v(1 / \kappa, \alpha)=0, \quad \text { for all } 1 / \kappa>\lambda_{0}^{-2} .
$$

That is, with ever improving work effectiveness $(1 / \kappa \nearrow \infty$ or $\alpha \searrow 2)$, the executive's fair compensation is ever increasing (to infinity). And with ever diminishing work effectiveness $\left(1 / \kappa \searrow \lambda_{0}^{-2}\right.$ or $\left.\alpha \nearrow \infty\right)$, the executive's work effort decreases towards base-level $\left(\lambda_{0}\right)$, for which the commensurate fair compensation is $v\left(e^{\lambda_{0}^{2} T / \alpha}-1\right)$; however, for the case where the executive becomes totally stressed $(\alpha \nearrow \infty)$, the fair compensation limit is zero.

\subsection{The Power-Utility Case}

Now with assumption of power-utility, the executive's optimal choice of work effort is $\lambda^{\star}(t)=\lambda_{0}^{\frac{\alpha}{\alpha-2}}((1 / \kappa) / \gamma)^{\frac{1}{\alpha-2}} f(t)^{\frac{1}{\alpha-2}}$ (see Theorem 3.1 for the optimal 
choice under the original disutility parameterization). To ensure optimal work effort is not less than base-level, we assume for the risk-free rate of return (in $f$ ) $r>-\lambda_{P}^{2} /(2 \gamma)$ (recalling that $\lambda_{P}$ is the Sharpe ratio of the market portfolio), and for work productivity

$$
1 / \kappa> \begin{cases}\gamma \lambda_{0}^{-2}, & \text { for } 0<\gamma<1 \\ \gamma \lambda_{0}^{-2} f(0)^{-1}, & \text { for } \gamma>1\end{cases}
$$

These conditions follow from the properties of the function $f$, which is the solution of a Bernoulli ODE (see Theorem 3.1 and its proof for $f$ and its derivation under the original disutility parameterization). Also note that $f(0)$ reads

$$
\begin{aligned}
f(0)= & e^{(1-\gamma)\left(r+\frac{1}{2} \frac{\lambda_{P}^{2}}{\gamma}\right) T} \\
& \times\left(1-\frac{(\alpha-2)\left(\frac{\lambda_{0}^{\alpha}}{\kappa \gamma}\right)^{\frac{2}{\alpha-2}}}{\alpha\left(2 \gamma r+\lambda_{P}^{2}\right)}\left(e^{\frac{1-\gamma}{\alpha-2}\left(2 r+\frac{\lambda_{P}^{2}}{\gamma}\right) T}-1\right)\right)^{-\frac{\alpha-2}{2}} .
\end{aligned}
$$

From (34), the executive's utility indifference (fair) up-front compensation $\Delta v$ is

$$
\begin{aligned}
\Delta v=v & \left(e^{\frac{1}{2 \gamma} \int_{0}^{T} \lambda^{\star}(t)^{2} \mathrm{~d} t}\right. \\
& \left.\times\left[1-\frac{(\alpha-2)\left(\frac{\lambda_{0}^{\alpha}}{\kappa \gamma}\right)^{\frac{2}{\alpha-2}}}{\alpha\left(2 \gamma r+\lambda_{P}^{2}\right)}\left(e^{\frac{1-\gamma}{\alpha-2}\left(2 r+\frac{\lambda_{P}^{2}}{\gamma}\right) T}-1\right)\right]^{\frac{(\alpha-2)}{2(1-\gamma)}}-1\right) .
\end{aligned}
$$

Remark 4.1 The solution presented for $\Delta v$ is derived using the structural properties of the executive's optimal personal investment strategy $\pi^{\star}$. An outside investor with knowledge of the work effort exercised by the executive (i.e. with knowledge of $\lambda^{\star}$ ) will choose a portfolio $\widehat{\pi}^{\star}$ identical to the executive's choice $\pi^{\star}$. Denote $\widehat{\Phi}(0, v)$ to be the maximized utility of the outside investor, then it follows that

$$
\widehat{\Phi}(0, v)=\Phi(0, v)+\mathbb{E}^{0, v}\left[\int_{0}^{T} c^{\star}\left(t, V_{t}^{\pi^{\star}}, \lambda^{\star}\left(t, V_{t}^{\pi^{\star}}\right)\right) \mathrm{d} t\right] .
$$


Furthermore

$$
\widehat{\Phi}(0, v)=\frac{v^{1-\gamma}}{1-\gamma} e^{(1-\gamma)\left[\left(r+\frac{1}{2} \frac{\lambda_{P}^{2}}{\gamma}\right) T+\frac{1}{2 \gamma} \int_{0}^{T} \lambda^{\star}(t)^{2} \mathrm{~d} t\right] .}
$$

Applying the utility indifference principle as given by (34), we can then solve $\widehat{\Phi}(0, v)=\Phi(0, v+\Delta v)$ to obtain $\Delta v$.

In contrast to the log-utility case, the sensitivities of the executive's optimal work effort $\lambda^{\star}$ and fair compensation $\Delta v$ with respect to variations in his work effectiveness parameters cannot be shown with compact expressions. Instead we limit ourselves to graphical representations of the relationships, with additional consideration of the executive's risk aversion parameter $\gamma$.

Figure 3 displays optimal work effort over time for varying risk aversion (i.e. $\lambda^{\star}$ versus $t$ and $\gamma$, for fixed values of $1 / \kappa, \alpha$ and $\lambda_{0}$ ). The executive's disutility from work effort depends on his wealth $v$ and risk aversion $\gamma$ via the scaling factor $v^{1-\gamma}$, which is effectively a work aversion measure. For a given level of wealth, an executive with low risk aversion $(0<\gamma<1)$ has higher work aversion than an executive with high risk aversion $(\gamma>$ 1); furthermore, with increasing wealth, work aversion increases for a low risk aversion executive but decreases for a high risk aversion executive. If we suggest that a high risk aversion executive has high work ethic and a low risk aversion executive has low work ethic, our set-up assumes that: a high work ethic executive has comparatively low aversion to work effort and will become further less averse to work effort if past effort or chance brings success as indicated by increased wealth; and a low work ethic executive has comparatively high aversion to work effort and will become further more averse to work effort if his wealth increases. Nevertheless, a low risk aversion (i.e. low work ethic) executive is more willing to take on the risk associated with a larger own-company stockholding, and thus with more personal stake in his own company he always applies more work effort than a high risk aversion executive, ceteris paribus. These aspects are observable in Figure 3: a low risk aversion executive with $0<\gamma<1$ starts with a (comparatively) high level of work effort, which is expected to reduce over time (given that his wealth is expected to increase over time); whereas a high risk aversion executive with $\gamma>1$ starts with a far lower level of work effort, which is expected to increase over time. Therefore observing the executive's work effort over time potentially reveals his risk aversion. 
Figures 4 and 5 fix the executive's risk aversion at a relatively low level of $\gamma=0.5$ and show optimal work effort over time for varying work effectiveness (i.e. respectively $\lambda^{\star}$ versus $t$ and $1 / \kappa$, and $\lambda^{\star}$ versus $t$ and $\alpha$ ). The executive's work effort increases with work effectiveness (but decreases over time given $\gamma=0.5)$. That is, work effort is positively related to work productivity $1 / \kappa$, and negatively related to disutility stress $\alpha$. The implication is that, for a given level of risk aversion, work effort distinguishes the work effectiveness (quality) of the executive.

The relationship between the executive's optimal work effort and his risk aversion and work effectiveness characteristics is reflected in his fair up-front compensation. Figures 6 and 7 show fair compensation versus pairings of risk aversion with each of work productivity and disutility stress (i.e. respectively $\Delta v$ versus $\gamma$ and $1 / \kappa$, and $\Delta v$ versus $\alpha$ and $\gamma$ ). Any combination of decreasing risk aversion, increasing work productivity, and decreasing disutility stress leads to higher work effort and commensurately higher fair compensation. The level of fair compensation is particularly prominently dependent on risk aversion: fair compensation sensitivity to work productivity and disutility stress is highest when risk aversion is low $(\gamma \approx 0.5$ or lower, see Figures 6 and 7). This result stems from the fact that, regardless of whether the executive has high work effectiveness or not, the company can only substantially benefit from the executive's quality if he has sufficiently low risk aversion to take on a substantial own-company stockholding and thereby have incentive to apply substantial work effort. Note that Figure 7 extends only to a minimum value of disutility stress $\alpha=5$; not shown is that for lower disutility stress $\alpha \approx 4$ and below, fair compensation increases even more steeply.

\section{Conclusion and Outlook}

We establish a model framework that gives insight into an unconstrained executive's own-company stockholding and work effort preferences. The executive's optimal work effort choice $\lambda^{\star}$ and fair compensation $\Delta v$ depend sensibly on his characteristics, risk aversion $\gamma$, work productivity $1 / \kappa$, and disutility stress $\alpha$. The executive's risk aversion is indicated by his work effort over time; and for a given level of risk aversion, the executive's work effectiveness quality (where higher quality is associated with higher work productivity and/or lower disutility stress) is distinguished by his work effort at a point in time. For empirical purposes, work effort might be observed with an 
empirical non-systematic Sharpe ratio or some other company performance measure.

We demonstrate that an executive with higher work effectiveness (quality) undertakes more work effort, which is associated with higher fair (utility indifference) compensation. Thus the executive is rewarded twice for his quality. First he receives higher compensation as a direct reward; and second he benefits from his work effort via his own-company stockholding, which can be considered an indirect reward.

The extent to which the company benefits from the executive's work effectiveness depends prominently on his risk aversion. Only if he has sufficiently low risk aversion to take on a substantial own-company stockholding will he have the incentive to apply substantial work effort for the benefit of the company. Consequently the executive's fair compensation is negatively related to his risk aversion.

Given identification of executive risk aversion and quality, our framework indicates the own-company stockholding and work effort of an unconstrained executive. This establishes a base case for theoretical or empirical assessment of the benefits or otherwise of constraining the executive with performance contracting. A future extension for our framework is to specify a constrained executive subject to an imposed own-company stockholding representative of performance contracting, and to contrast his work effort strategy with that of our unconstrained executive.

\section{Appendix}

Proof of Lemma 2.1. Fix $(t, v, \lambda) \in[0, T] \times \mathbb{R}^{+} \times \mathbb{R}_{0}^{+}$and define the function $f$ by $f(\sigma)=c(t, v, r+\lambda \sigma, \sigma)$, for $\lambda \geq 0$. We need to show for $f$ that a minimizing $\sigma^{\star}=\sigma^{\star}(t, v, \lambda)$ exists and is unique. Computing the first and second derivatives gives

$$
f^{\prime}(\sigma)=\lambda \frac{\partial c}{\partial \mu}(t, v, r+\lambda \sigma, \sigma)+\frac{\partial c}{\partial \sigma}(t, v, r+\lambda \sigma, \sigma),
$$

and

$f^{\prime \prime}(\sigma)=\lambda^{2} \frac{\partial^{2} c}{\partial \mu^{2}}(t, v, r+\lambda \sigma, \sigma)+2 \lambda \frac{\partial^{2} c}{\partial \sigma \partial \mu}(t, v, r+\lambda \sigma, \sigma)+\frac{\partial^{2} c}{\partial \sigma^{2}}(t, v, r+\lambda \sigma, \sigma)$.

By the differentiability assumption for $c, f^{\prime}$ is continuous and differentiable and $f^{\prime \prime}$ is continuous. Using elementary calculus rationale, the minimization 
problem $\min _{\sigma>0} f(\sigma)$ admits a unique solution if $f^{\prime}\left(\sigma^{\star}\right)=0$ has a solution and $f$ is strictly convex.

For $f^{\prime}\left(\sigma^{\star}\right)=0$ to admit a solution that locally minimizes $f$, it is sufficient that $f^{\prime}$ starts below zero, $f^{\prime}(0+)<0$, and that $f^{\prime}$ takes on a positive value for some $\sigma>0+$. This is given by Assumption 2.1 (ii). Moreover the condition $f$ is strictly convex, $f^{\prime \prime}>0$, implies the solution is a unique global minimizer. Assumption 2.1 (iii) gives the strict convexity of $f$.

Proof of Theorem 3.3. Define the performance functional of our optimal investment and control decision by

$$
J(t, v ; \pi, \lambda):=\mathbb{E}^{t, v}\left[U\left(V_{T}^{\pi}\right)-\int_{t}^{T} c^{\star}\left(u, V_{u}^{\pi}, \lambda_{u}\right) \mathrm{d} u\right],
$$

where $(t, v) \in[0, T] \times \mathbb{R}^{+}$and $(\pi, \lambda) \in A_{\gamma}^{\prime}(t, v)$. Recall the claimed optimal value function $\Phi \in C^{1,2}$, for $\gamma>0$, and apply Ito's formula to obtain:

$$
\begin{aligned}
& U\left(V_{T}^{\pi}\right)-\int_{t}^{T} c^{\star}\left(u, V_{u}^{\pi}, \lambda_{u}\right) \mathrm{d} u=\Phi\left(T, V_{T}^{\pi}\right)-\int_{t}^{T} \kappa\left(V_{u}^{\pi}\right)^{1-\gamma} \frac{\lambda_{u}^{\alpha}}{\alpha} \mathrm{d} u=\Phi(t, v) \\
& +\int_{t}^{T}\left(\Phi_{t}\left(u, V_{u}^{\pi}\right)+\Phi_{v}\left(u, V_{u}^{\pi}\right) V_{u}^{\pi}\left[r+\pi_{u}^{S} \lambda \sigma_{u}^{\star}+\left(\pi_{u}^{P}+\beta \pi_{u}^{S}\right)\left(\mu^{P}-r\right)\right]\right. \\
& \left.+1 / 2 \Phi_{v v}\left(u, V_{u}^{\pi}\right)\left(V_{u}^{\pi}\right)^{2}\left[\left(\left(\pi^{P}+\beta \pi_{u}^{S}\right) \sigma^{P}\right)^{2}+\left(\pi_{u}^{S} \sigma_{u}^{\star}\right)^{2}\right]-\kappa\left(V_{u}^{\pi}\right)^{1-\gamma} \frac{\lambda_{u}^{\alpha}}{\alpha}\right) \mathrm{d} u \\
& +\int_{t}^{T} \Phi_{v}\left(u, V_{u}^{\pi}\right) V_{u}^{\pi}\left(\pi_{u}^{P}+\beta \pi_{u}^{S}\right) \sigma^{P} \mathrm{~d} W_{u}^{P}+\int_{t}^{T} \Phi_{v}\left(u, V_{u}^{\pi}\right) V_{u}^{\pi} \pi_{u}^{S} \sigma_{u}^{\star} \mathrm{d} W_{u} .
\end{aligned}
$$

The remainder of the proof is divided into two parts. Part (a) establishes that the value function $\Phi$ coincides with the performance functional $J$ evaluated at the claimed maximizers $\left(\pi^{\star}, \lambda^{\star}\right), \gamma>0$. Part (b) shows the optimality of the candidate $\left(\pi^{\star}, \lambda^{\star}\right)$, i.e.: $J(t, v ; \pi, \lambda) \leq \Phi(t, v)$, for $(\pi, \lambda) \in A_{\gamma}^{\prime}(t, v)$.

Part (a): We establish that $J\left(t, v ; \pi^{\star}, \lambda^{\star}\right)=\Phi(t, v)$. To do this we show that in the right hand side (RHS) of (35) the drift vanishes by the HJB (19) and that the local martingale component is a true martingale and hence disappears in expectation. And finally, it is verified that indeed $\left(\pi^{\star}, \lambda^{\star}\right) \in$ $A_{\gamma}^{\prime}(t, v)$.

By construction, $\Phi$ with control $\left(\pi^{\star}, \lambda^{\star}\right)$ satisfies the HJB-PDE in (19), 
that is,

$$
\begin{aligned}
0= & \Phi_{t}+\Phi_{v} v\left(r+\pi^{S \star} \lambda^{\star} \sigma^{\star}+\left(\pi^{P \star}+\beta \pi^{S \star}\right)\left[\mu^{P}-r\right]\right) \\
& +(1 / 2) \Phi_{v v} v^{2}\left(\left[\pi^{S \star} \sigma^{\star}\right]^{2}+\left[\left(\pi^{P \star}+\beta \pi^{S \star}\right) \sigma^{P}\right]^{2}\right)-c^{\star} .
\end{aligned}
$$

This eliminates the drift (Lebesgue integral) in (35) and we obtain

$$
\begin{aligned}
& U\left(V_{T}^{\pi^{\star}}\right)-\int_{t}^{T} c^{\star}\left(u, V_{u}^{\pi^{\star}}, \lambda_{u}^{\star}\right) \mathrm{d} u=\Phi(t, v)+ \\
& \int_{t}^{T} \Phi_{v}\left(u, V_{u}^{\pi^{\star}}\right) V_{u}^{\pi^{\star}}\left(\pi_{u}^{P \star}+\beta \pi_{u}^{S \star}\right) \sigma^{P} \mathrm{~d} W_{u}^{P}+\int_{t}^{T} \Phi_{v}\left(u, V_{u}^{\pi^{\star}}\right) V_{u}^{\pi^{\star}} \pi_{u}^{S \star} \sigma_{u}^{\star} \mathrm{d} W_{u} .
\end{aligned}
$$

For $J\left(t, v ; \pi^{\star}, \lambda^{\star}\right)=\Phi(t, v)$, it remains to prove that the local martingale component disappears in expectation. A sufficient condition is the squareintegrability of the local martingale component

$$
\mathbb{E}\left[\int_{t}^{T}\left(\Phi_{v}\left(u, V_{u}^{\pi^{\star}}\right) V_{u}^{\pi^{\star}}\right)^{2}\left(\left[\pi_{u}^{P^{\star}}+\beta \pi_{u}^{S \star}\right]^{2}\left(\sigma^{P}\right)^{2}+\left[\pi_{u}^{S^{\star}} \sigma_{u}^{\star}\right]^{2}\right) \mathrm{d} u\right]<\infty .
$$

Using the explicit form of the candidates in (24), for $\gamma>0$ and $\gamma \neq 1$, and in $(31)$, for $\gamma=1$, gives

$$
\begin{aligned}
\left(\Phi_{v}\left(u, V_{u}^{\pi^{\star}}\right) V_{u}^{\pi^{\star}}\right)^{2}( & {\left.\left[\pi_{u}^{P^{\star}}+\beta \pi_{u}^{S \star}\right]^{2}\left(\sigma^{P}\right)^{2}+\left[\pi_{u}^{S^{\star}} \sigma_{u}^{\star}\right]^{2}\right) } \\
= & \frac{\left(V_{u}^{\pi^{\star}}\right)^{2(1-\gamma)} f(u)^{2}}{\gamma^{2}}\left[\frac{\left(\mu^{P}-r\right)^{2}}{\left(\sigma^{P}\right)^{2}}+\left(\frac{1}{\kappa \gamma} f(u)\right)^{\frac{2}{\alpha-2}}\right]
\end{aligned}
$$

where we set $f=1$, for $\gamma=1$. The RHS is $\left(V_{u}^{\pi^{\star}}\right)^{2(1-\gamma)}$ times a deterministic and continuous function on the compact set $[t, T]$. The deterministic part is uniformly bounded. Therefore, it is sufficient to focus on the stochastic component: $V^{\pi^{\star}}$ satisfies

$\mathrm{d} V_{u}^{\pi^{\star}}=V_{u}^{\pi^{\star}}\left[r \mathrm{~d} u+\frac{\lambda_{P}^{2}}{\gamma} \mathrm{d} u+\frac{\left(\lambda^{\star}\left(u, V_{u}^{\pi^{\star}}\right)\right)^{2}}{\gamma} \mathrm{d} u+\frac{\lambda_{P}}{\gamma} \mathrm{d} W_{u}^{P}+\frac{\lambda^{\star}\left(u, V_{u}^{\pi^{\star}}\right)}{\gamma} \mathrm{d} W_{u}\right]$.

Recalling that $\lambda^{\star}(u, v)$ is a continuous function in $u$ and does not depend on $v$, we see that $V_{u}^{\pi^{\star}}$ follows a log-normal distribution with parameters being uniformly bounded, for all $u \in[t, T]$. Since all moments of a log-normally distributed random variable exist, it follows that the local martingale is a 
square-integrable martingale. This establishes $J\left(t, v ; \pi^{\star}, \lambda^{\star}\right)=\Phi(t, v)$. Finally, $\left(\pi^{\star}, \lambda^{\star}\right) \in A_{\gamma}^{\prime}(t, v)$ follows from the fact that $\pi^{P \star}, \pi^{S \star} \sigma^{\star}$, and $\lambda^{\star}$ are uniformly bounded on $[t, T]$, each $\gamma>0$.

Part (b): Now we show the optimality, i.e. $J(t, v ; \pi, \lambda) \leq \Phi(t, v)$, for $(\pi, \lambda) \in A_{\gamma}^{\prime}(t, v)$. As in (a), this is also based on the analysis of (35). The HJB (19) is applied to show that the drift component is bounded from above by zero. Then it is shown that the conditions in Def. 3.1 are sufficient for the local martingale component on the RHS of (35) to vanish in expectation.

By the HJB (19), $\Phi$ with arbitrary $(\pi, \lambda) \in \mathbb{R} \times \mathbb{R} \times \mathbb{R}_{0}^{+}$satisfies

$$
\begin{aligned}
0 \geq & \Phi_{t}+\Phi_{v} v\left(r+\pi^{S} \lambda \sigma^{\star}+\left(\pi^{P}+\beta \pi^{S}\right)\left[\mu^{P}-r\right]\right) \\
& +(1 / 2) \Phi_{v v} v^{2}\left(\left[\pi^{S} \sigma^{\star}\right]^{2}+\left[\left(\pi^{P}+\beta \pi^{S}\right) \sigma^{P}\right]^{2}\right)-c^{\star},
\end{aligned}
$$

for $(u, v) \in[t, T] \times \mathbb{R}^{+}$. This provides the point-wise upper bound zero for the drift in (35) and we obtain

$$
\begin{aligned}
& U\left(V_{T}^{\pi}\right)-\int_{t}^{T} c^{\star}\left(u, V_{u}^{\pi}, \lambda_{u}\right) \mathrm{d} u \leq \Phi(t, v)+ \\
& \underbrace{\int_{t}^{T} \Phi_{v}\left(u, V_{u}^{\pi}\right) V_{u}^{\pi}\left(\pi_{u}^{P}+\beta \pi_{u}^{S}\right) \sigma^{P} \mathrm{~d} W_{u}^{P}+\int_{t}^{T} \Phi_{v}\left(u, V_{u}^{\pi}\right) V_{u}^{\pi} \pi_{u}^{S} \sigma^{\star}\left(u, V_{u}^{\pi}, \lambda_{u}\right) \mathrm{d} W_{u}}_{=: M_{T}^{t}} .
\end{aligned}
$$

We discuss two separate cases: (b1): $0<\gamma<1$ and $\gamma>1$, and (b2): $\gamma=1$.

Part (b1): $0<\gamma<1$ and $\gamma>1$. Recall $\Phi_{v}(t, v)=f(t) v^{-\gamma}$ and calculate the quadratic variation of $M^{t}$

$$
\begin{aligned}
& \left\langle M^{t}\right\rangle_{T}=\int_{t}^{T}\left(V_{u}^{\pi}\right)^{2(1-\gamma)} f^{2}(u)\left(\left[\pi_{u}+\beta \pi_{u}^{S}\right]^{2}\left(\sigma^{P}\right)^{2}+\left[\sigma_{u}^{\star} \pi_{u}^{S}\right]^{2}\right) \mathrm{d} u \\
& \leq \frac{1}{2} \sup _{0 \leq u \leq T} f(u)^{2}\left[\int_{t}^{T}\left(V_{u}^{\pi}\right)^{4(1-\gamma)} \mathrm{d} u+\int_{t}^{T}\left(\left[\pi_{u}+\beta \pi_{u}^{S}\right]^{2}\left(\sigma^{P}\right)^{2}+\left[\sigma_{u}^{\star} \pi_{u}^{S}\right]^{2}\right)^{2} \mathrm{~d} u\right]
\end{aligned}
$$

where the second line is a straightforward upper bound. We show that $M^{t}$ is a martingale by deriving the integrability of the quadratic variation $\left\langle M^{t}\right\rangle_{T}$. First we use once more that $f$ is a continuous function on the compact set $[0, T]$ and is uniformly bounded, and thus $\sup _{0 \leq u \leq T} f(u)^{2}$ is finite. We are left to deal with the two expressions in the brackets of (37). The second 
expression is bounded in expectation by assumption, see (15) in Def. 3.1. In what follows we establish that the first expression is finite by showing that $\mathbb{E}^{t, v}\left[\left(V_{u}^{\pi}\right)^{\xi}\right]<\infty$ uniformly, with $\xi=4(1-\gamma)$, where $\xi>0$ for $0<\gamma<1$ and $\xi<0$ for $\gamma>1$.

The solution of the wealth equation (4) starting at $t$ with initial wealth $v$ is

$$
V_{u}^{\pi}=v e^{r(u-t)+\int_{t}^{u}\left(\pi_{s}^{P}+\beta \pi_{s}^{S}\right) \lambda^{P} \sigma^{P}+\pi_{s}^{S} \lambda_{s} \sigma_{s}^{\star} \mathrm{d} s} e^{L_{u}^{t}-\frac{1}{2}\left\langle L^{t}\right\rangle_{u},}
$$

where $L_{u}^{t}=\int_{t}^{u}\left(\pi_{s}^{P}+\beta \pi_{s}^{S}\right) \sigma^{P} \mathrm{~d} W_{s}^{P}+\int_{t}^{u} \pi_{s}^{S} \sigma_{s}^{\star} \mathrm{d} W_{s}$ and $\left\langle L^{t}\right\rangle_{u}=\int_{t}^{u}\left(\pi_{s}^{P}+\right.$ $\left.\beta \pi_{s}^{S}\right)^{2}\left(\sigma^{P}\right)^{2}+\left(\pi_{s}^{S} \sigma_{s}^{\star}\right)^{2} \mathrm{~d} s$. Then

$$
\left(V_{u}^{\pi}\right)^{\xi}=v^{\xi} e^{\xi L_{u}^{t}-\frac{1}{2} \xi^{2}\left\langle L^{t}\right\rangle_{u}} \times e^{\xi\left[\frac{1}{2}(\xi-1)\left\langle L^{t}\right\rangle_{u}+r(u-t)+\int_{t}^{u}\left(\pi_{s}^{P}+\beta \pi_{s}^{S}\right) \lambda^{P} \sigma^{P}+\pi_{s}^{S} \lambda_{s} \sigma_{s}^{\star} \mathrm{d} s\right]} .
$$

The second factor is uniformly bounded by a constant, see Def. 3.1, (15) and (16), and recalling that $\xi>0$ for $0<\gamma<1$ and $\xi<0$ for $\gamma>1$. It remains to prove that the first factor $Z_{u}^{t}=e^{\xi L_{u}^{t}-\frac{1}{2} \xi^{2}\left\langle L^{t}\right\rangle_{u}}, t \leq u \leq T$, is integrable. However, $Z^{t}$ is a strictly positive local martingale since it is the stochastic exponential of the local martingale $\xi L^{t}$. The Novikov condition holds by (15), i.e. $\mathbb{E}^{t, v}\left(e^{\frac{1}{2} \xi^{2}\left\langle L^{t}\right\rangle_{T}}\right)<\infty$, and hence $Z^{t}$ is a true martingale and $\mathbb{E}^{t, v}\left(Z_{u}^{t}\right)=1, t \leq u \leq T$. The local martingale $M^{t}$ is therefore a martingale vanishing in expectation in (36), and taking the conditional expectation of (36) gives the desired result

$J(t, v ; \pi, \lambda)=\mathbb{E}^{t, v}\left[U\left(V_{T}^{\pi}\right)-\int_{t}^{T} c^{\star}\left(u, V_{u}^{\pi}, \lambda_{u}\right) \mathrm{d} u\right] \leq \Phi(t, v),(\pi, \lambda) \in A_{\gamma}^{\prime}(t, v)$.

Part (b2): $\gamma=1$. From $\Phi_{v}(t, v)=v^{-1}$ we obtain

$$
M_{s}^{t}=\int_{t}^{s}\left(\pi_{u}^{P}+\beta \pi_{u}^{S}\right) \sigma^{P} \mathrm{~d} W_{u}^{P}+\int_{t}^{s} \pi_{u}^{S} \sigma^{\star}\left(u, V_{u}^{\pi}, \lambda_{u}\right) \mathrm{d} W_{u}, \quad \text { for } t \leq s \leq T .
$$

Def. 3.1 (ii) ensures the square-integrability. The local martingale $M^{t}$ is therefore a martingale vanishing in expectation in $(36)$, and $J(t, v ; \pi, \lambda) \leq$ $\Phi(t, v)$, for $(\pi, \lambda) \in A_{1}^{\prime}(t, v)$. 


\section{References}

Aseff J, Bizjak J, Lemmon M (2001) Managerial ownership, incentive contracting, and the use of zero-cost collars and equity swaps by corporate insiders. J of Financial and Quantitative Analysis 36(3):345-370

Cadenillas A, Cvitanić J, Zapatero F (2004) Leverage decision and manager compensation with choice of effort and volatility. J of Financial Economics $73(1): 71-92$

Carpenter J (2000) Does option compensation increase managerial risk appetite? J of Finance 55(5):2311-2331

Core J, Larcker D (2002) Performance consequences of mandatory increases in executive stock ownership. J of Financial Economics 64(3):317-340

Core J, Guay W, Larcker D (2003) Executive equity compensation and incentives: A survey. Economic Policy Rev 9:27-50

Cvitanić J (2008) On managerial risk-taking incentives when compensation may be hedged against. Working Paper, Caltech, Pasadena

Dittmann I, Maug E (2007) Lower salaries and no options? On the optimal structure of executive pay. J of Finance 62(1):303-343

Garvey G, Milbourn T (2003) Incentive compensation when executives can hedge the market: Evidence of relative performance evaluation in the cross section. J of Finance 58(4):1557-1581

Hall B, Liebman J (1998) Are CEOs really paid like bureaucrats? Q J of Economics 113(3):653-691

Holmstrom B (1979) Moral hazard and observability. Bell J of Economics 10:74-91

Jensen M, Meckling W (1976) Theory of the firm: Managerial behavior, agency costs and ownership structure. J of Financial Economics 3(4):305360

Jensen M, Murphy K (1990) Performance pay and top-management incentives. J of Political Economics 98(2):225-264 
Jin L (2002) CEO compensation, diversification and incentives. J of Financial Economics 66(1):29-63

Korn R, Korn E (2001) Option pricing and portfolio optimization. Graduate Studies in Mathematics, Volume 31, American Mathematical Society

Korn R, Kraft H (2008) Continuous-time delegated portfolio management with homogeneous expectations: Can an agency conflict be avoided? Financial Markets and Portf Management 22(1):67-90

Lambert R, Larcker D, Verrecchia R (1991) Portfolio considerations in valuing executive compensation. J of Account Res 29(1):129-148

Murphy K (1999) Executive compensation. Ashenfelter and D. Card, eds., Handbook of labor economics, Vol. 3, Amsterdam, North-Holland.

Ofek E, Yermack D (2000) Taking stock: Equity-based compensation and the evolution of managerial ownership. J of Finance 55(3):1367-1384

Ou-Yang H (2003) Optimal contracts in a continuous-time delegated portfolio management problem. Rev of Financial Stud 16(1):173-208

Ross S (1973) The economic theory of agency: The principal's problem. American Economic Rev 63(2):134-139

Ross S (2004) Compensation, incentives, and the duality of risk aversion and riskiness. J of Finance 59(1):207-225 


\section{Figures}

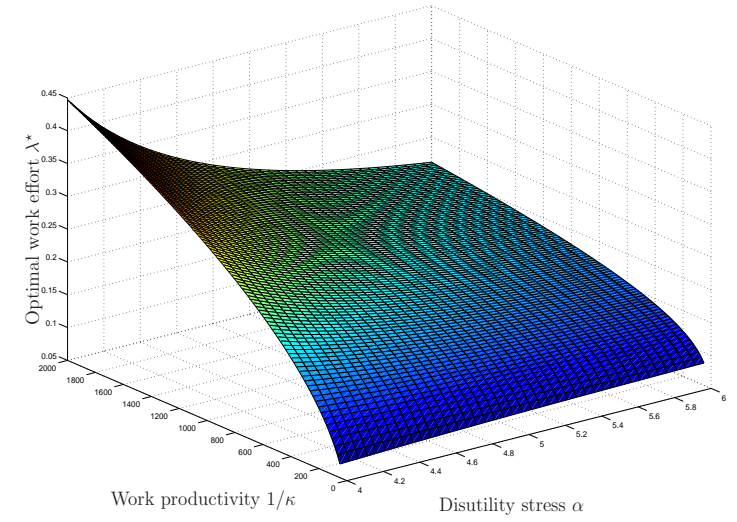

Figure 1: The log-utility executive's optimal work effort/control choice, in terms of optimal non-systematic Sharpe ratio $\lambda^{\star}$, versus his work effectiveness parameters, work productivity $1 / \kappa$ and disutility stress $\alpha$; given base-level work effort $\lambda_{0}=0.10$.

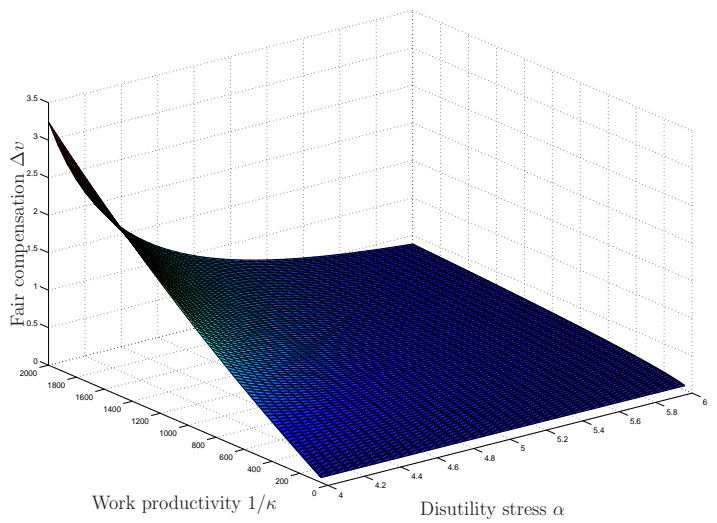

Figure 2: The log-utility executive's fair up-front compensation $\Delta v$, based on utility indifference, versus his work effectiveness parameters, work productivity $1 / \kappa$ and disutility stress $\alpha$; given initial wealth $v=\$ 5$ million, time horizon $T=10$ years, and base-level work effort $\lambda_{0}=0.10$. 


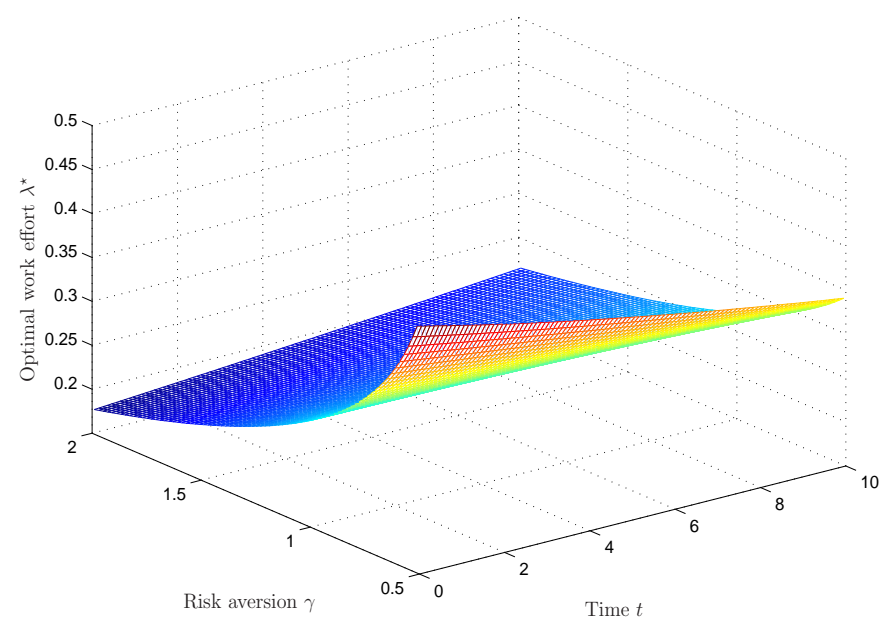

Figure 3: The power-utility executive's optimal work effort/control choice, in terms of optimal non-systematic Sharpe ratio $\lambda^{\star}$, versus time $t$, for varying risk-aversion $\gamma$; given work productivity $1 / \kappa=2000$, disutility stress $\alpha=5$, and base-level work effort $\lambda_{0}=0.10$.

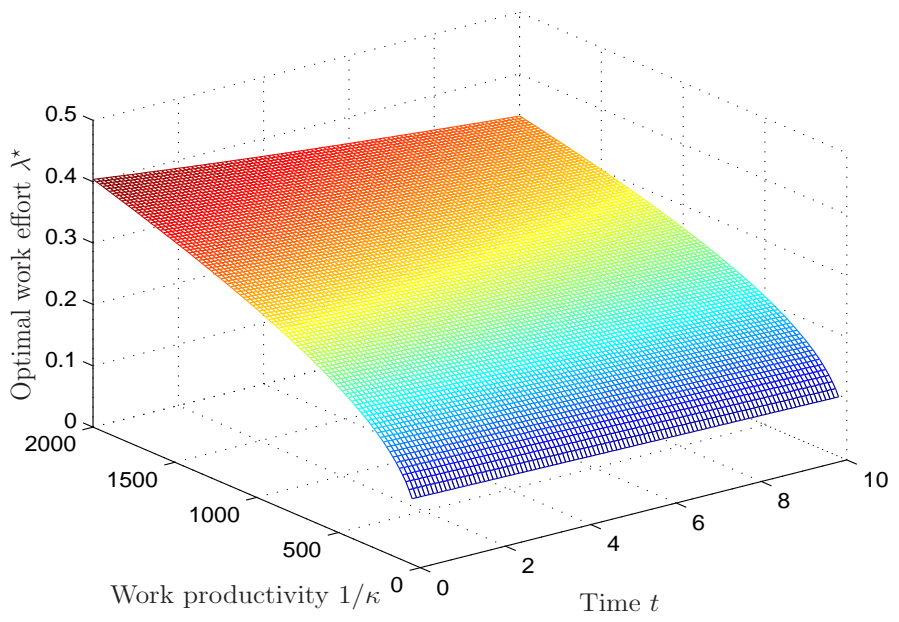

Figure 4: The power-utility executive's optimal work effort/control choice, in terms of optimal non-systematic Sharpe ratio $\lambda^{\star}$, versus time $t$, for varying work productivity $1 / \kappa$; given risk aversion $\gamma=0.5$, disutility stress $\alpha=5$, and base-level work effort $\lambda_{0}=0.10$. 


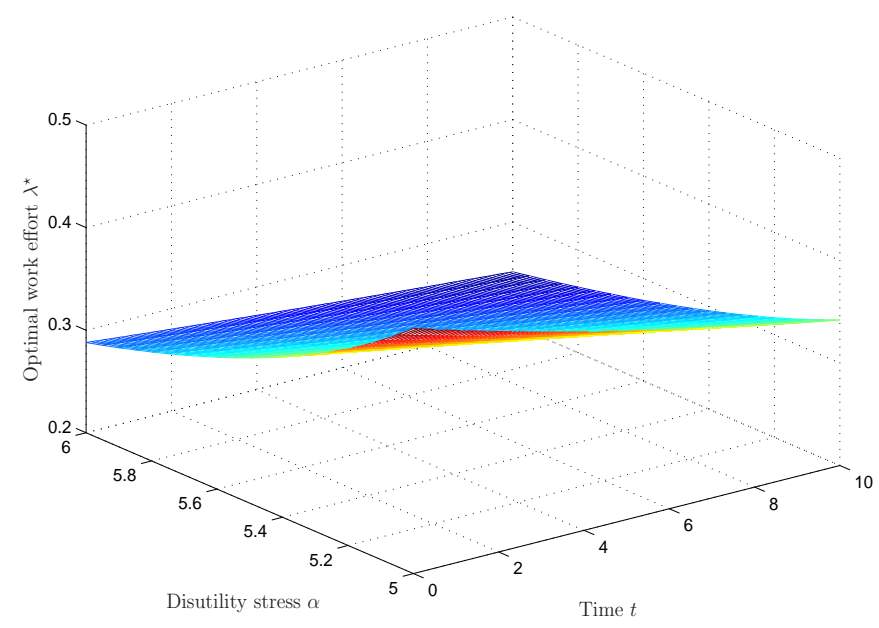

Figure 5: The power-utility executive's optimal work effort/control choice, in terms of optimal non-systematic Sharpe ratio $\lambda^{\star}$, versus time $t$, for varying disutility stress $\alpha$; given risk aversion $\gamma=0.5$, work productivity $1 / \kappa=2000$, and base-level work effort $\lambda_{0}=0.10$.

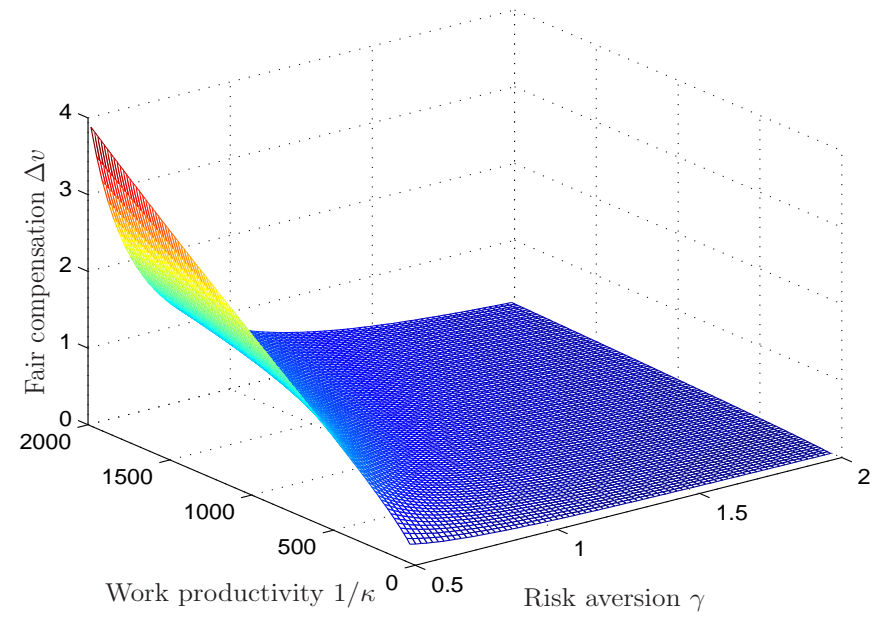

Figure 6: The power-utility executive's fair up-front compensation $\Delta v$, based on utility indifference, versus his work productivity $1 / \kappa$ and risk aversion $\gamma$; given disutility stress $\alpha=5$, initial wealth $v=\$ 5$ million, time horizon $T=10$ years, and base-level work effort $\lambda_{0}=0.10$. 


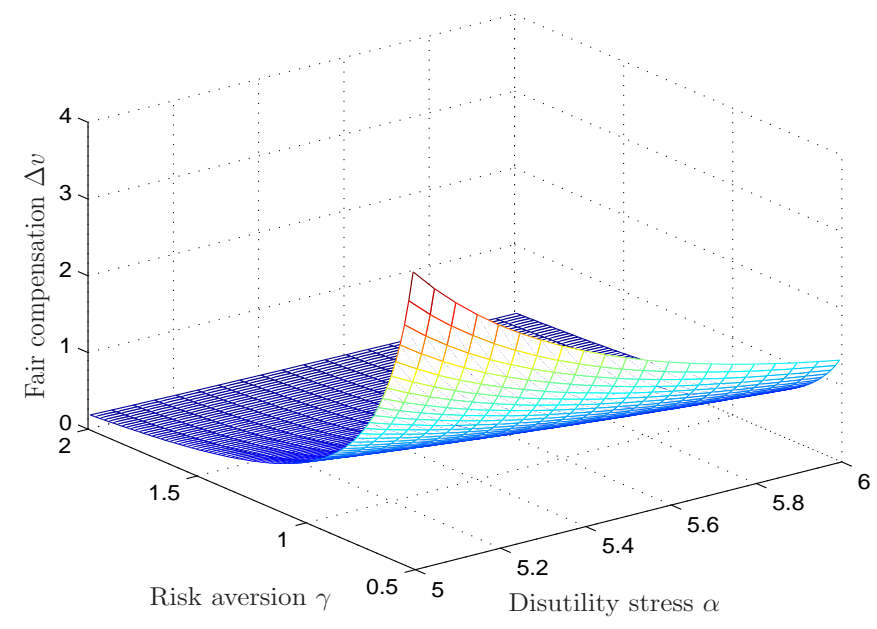

Figure 7: The power-utility executive's fair up-front compensation $\Delta v$, based on utility indifference, versus his risk aversion $\gamma$ and disutility stress $\alpha$; given work productivity $1 / \kappa=2000$, initial wealth $v=\$ 5$ million, time horizon $T=10$ years, and base-level work effort $\lambda_{0}=0.10$. 